\title{
AN APPROXIMATE NONLINEAR PROJECTION SCHEME FOR A COMBUSTION MODEL
}

\author{
Christophe Berthon $^{1,2}$ And Didier Reignier ${ }^{1}$
}

\begin{abstract}
The paper deals with the numerical resolution of the convection-diffusion system which arises when modeling combustion for turbulent flow. The considered model is of compressible turbulent reacting type where the turbulence-chemistry interactions are governed by additional balance equations. The system of PDE's, that governs such a model, turns out to be in non-conservation form and usual numerical approaches grossly fail in the capture of viscous shock layers. Put in other words, classical finite volume methods induce large errors when approximated the convection-diffusion extracted system. To solve this difficulty, recent works propose a nonlinear projection scheme based on cancellation phenomenon of relevant dissipation rates of entropy. Unfortunately, such a property never holds in the present framework. The nonlinear projection procedures are thus extended.
\end{abstract}

Mathematics Subject Classification. 65M99, 76L05, 76M25.

Received: May 14, 2002. Revised: February 24, 2003.

\section{INTRODUCTION}

An accurate description of the effects of turbulence-chemistry interactions is required to predict most reacting flows of practical interest. In the present work, we are interested in the numerical resolution of a convectiondiffusion system which arises when modeling combustion for turbulent flow. We consider here a model where the combustion is modeled with two passive scalars. Both scalars are governed by "transport" equations. Unfortunately, the system does not recast in conservation form excepted for restrictive modeling assumptions. The numerical capture of the viscous shock layers coming with considered nonconservative PDE's systems, is the topic of the present work. Since the Reynolds numbers of interest are large, these layers display the character of a shock wave in that they differ from their end states only in a small interval of rapid transition (see BerthonCoquel [3], Raviart-Sainsaulieu [21]). Hence for mesh refinements of practical interest, the associated discrete profiles stay largely under resolved. Our purpose is actually to correctly capture the two end states, $\mathbf{v}_{L}$ and $\mathbf{v}_{R}$, of a given shock layer together with its relevant speed of propagation $\sigma$ without resolving sharply the viscous layer itself.

Keywords and phrases. Hyperbolic systems in nonconservation form, finite volume methods, nonlinear projection method.

1 MAB UMR 5466 CNRS, Université Bordeaux I, 351 cours de la libération, 33400 Talence, France.

2 INRIA Futurs, Domaine de Voluceau-Rocquencourt, BP 105, 78153 Le Chesnay Cedex, France.

e-mail: christophe.berthon@math.u-bordeaux.fr 
It is quite well-known that such an issue does not raise special difficulties within the standard frame of the Navier-Stokes equations, e.g. in conservation form. Indeed, the triples $\left(\sigma ; \mathbf{v}_{L}, \mathbf{v}_{R}\right)$ are solutions of the classical Rankine-Hugoniot relations and finite volume methods in conservation form readily ensure their suitable capture.

The situation turns out to be completely different in the setting of the non conservation model. Its non conservation form makes this time the Rankine-Hugoniot relations unknown (see Dal Maso-LeFloch-Murat [11], LeFloch [19]). A close characterization of the triples $\left(\sigma ; \mathbf{v}_{L}, \mathbf{v}_{R}\right)$ is proposed on the basis of generalized jump relations which involve the entropy dissipation rates. In fact, the dissipation rates of entropy do not evolve independently but proportionally.

Underlining that despite relevant Godunov type methods actually enjoy several stability properties (see Berthon-Coquel [5], Godlewski-Raviart [13]), their corresponding numerical rate of entropy dissipation stays always smaller than the required one and as a result, the discrepancies with the exact solutions can only amplify with time. This error analysis will then suggest the introduction of a nonlinear projection step that enforce the validity of the generalized jump conditions at the discrete level [5]. In the framework of compressible turbulent flows (for instance the $(k, \epsilon)$ model), such a nonlinear projection gives a good agreement between exact and discrete solutions. Furthermore, approximate solutions performed thanks to the nonlinear method satisfy the required positivity preserving properties in addition to several (nonlinear) stability requirements.

In the present paper, the combustion turbulent model we are focusing on differs from the usual $(k, \epsilon)$ model by two additional unknowns associated with combustion phenomena and governed by transport equations with production terms. As in [5], these production terms play a crucial role when approximating viscous shock layers. The main difference with the standard $(k, \epsilon)$ model stays in the lack of proportionality relations between the production terms. In the framework of large Reynolds numbers, we establish that the production terms are almost proportional with an error in $O(1 / R e)$. Then, we deduce an approximate nonlinear projection procedure.

The present paper is organized as follows. In the next section, we introduce the set of equations and we state algebraic properties satisfied by the solutions. Next, we emphasize the numerical negative consequences of the non-conservation form of the system. In the third section, we explain the discrete scheme; as a first step we recall the nonlinear projection scheme (see [5]) and as a second step we develop a new nonlinear procedure according to our system. In the last section, some numerical results are given.

\section{THE PHYSICAL MODEL}

An accurate description of the effects of turbulence-chemistry interactions is required to predict most reacting flows of practical interest. These effects play an important role in a number of industrial applications where physico-chemical phenomena are crucial. The considered model is devoted to multicomponent turbulent reactive flow (see Baurle and Girimaji [2], Laborde [16] for similar model). Three distinct interacting physical phenomena are considered, namely the flow of the fluid, the turbulence and the combustion. The flow is characterized by the total density $\rho>0$, the mass fraction of each component $C_{j} \in[0,1]$ with $1 \leq j \leq N$, where $N \geq 2$, the velocity vector $U \in \mathbb{R}^{3}$ and the total energy $E>0$. Concerning the turbulence, the standard $(k, \epsilon)$ model is adopted (see Berthon and Coquel [4], but also Larrouturou and Olivier [18], Mohammadi and Pironneau [20]). The turbulence is thus governed by the kinetic turbulent energy $k>0$ and its dissipation rate $\epsilon>0$. Following Laborde [16], the combustion is modeled with two passive scalars namely $k_{h}>0$ the enthalpy variance and $Q>0$ the variance of mass fractions. Let us note that the introduction of passive scalars is generally used as soon as algebraic closures are not prescribed by the modeling assumptions. The considered $(k, \epsilon, g)$ model, where $g$ denotes the passive scalars, is governed by a convection-diffusion-reactive PDE system. Owing to the rotational invariance of the model of interest, one space dimension is assumed with no restriction to obtain the 
following system:

$$
\begin{aligned}
& \left\{\begin{array}{l}
\partial_{t} \rho C_{j}+\partial_{x} \rho C_{j} u=\partial_{x}\left(\left(\rho D+\rho D_{t}\right) \partial_{x} C_{j}\right)+\overline{\dot{\omega}_{j}}, \quad 1 \leq j \leq N, \sum_{1 \leq j \leq N} C_{j}=1, \\
\partial_{t} \rho u+\partial_{x}\left(\rho u^{2}+p+\frac{2}{3} \rho k\right)=\partial_{x}\left(\left(\mu+\mu_{t}\right) \partial_{x} u\right), \\
\partial_{t} E+\partial_{x}\left(E+p+\frac{2}{3} \rho k\right) u=\partial_{x}\left(\left(\mu+\mu_{t}\right) u \partial_{x} u\right)-\partial_{x} q+\partial_{x}\left(\mu_{k} \partial_{x} k\right),
\end{array}\right. \\
& \left\{\begin{array}{l}
\partial_{t} \rho k+\partial_{x} \rho k u+\frac{2}{3} \rho k \partial_{x} u=\mu_{t}\left(\partial_{x} u\right)^{2}+\partial_{x}\left(\mu_{k} \partial_{x} k\right)-\rho \epsilon \\
\partial_{t} \rho \epsilon+\partial_{x} \rho \epsilon u+\frac{2}{3} C_{\epsilon 1} \rho \epsilon \partial_{x} u=C_{\epsilon 1} \frac{\epsilon}{k} \mu_{t}\left(\partial_{x} u\right)^{2}+\partial_{x}\left(\mu_{\epsilon} \partial_{x} \epsilon\right)-C_{\epsilon 2} \rho \frac{\epsilon^{2}}{k}
\end{array}\right. \\
& \left\{\begin{array}{l}
\partial_{t} \rho k_{h}+\partial_{x} \rho k_{h} u=\partial_{x}\left(\frac{\lambda_{t}}{C_{p}} \partial_{x} k_{h}\right)+2 \frac{\lambda_{t}}{C_{p}}\left(\partial_{x} p^{\star}\right)^{2}-2 \rho C_{g 1} \frac{\epsilon}{k} k_{h}, \\
\partial_{t} \rho Q+\partial_{x} \rho Q u=\partial_{x}\left(\rho D_{t} \partial_{x} Q\right)+2 \frac{\lambda_{t}}{S_{c t}} \sum_{1 \leq j \leq N}\left(\partial_{x} C_{j}\right)^{2}-2 \rho C_{g 2} \frac{\epsilon}{k} Q+2 \sum_{1 \leq j \leq N} \overline{\dot{\omega}_{j} C_{j}^{\prime \prime}} .
\end{array}\right.
\end{aligned}
$$

We refer to Baurle and Girimaji [2] or Laborde [16] for the details of the functions and constants involved in the system. Briefly, we recall that $\mu>0$ and $\mu_{t}>0$ respectively denote the laminar and turbulent viscosity functions: $\mu$ is given by the Sutherland law while we set

$$
\mu_{t}=C_{\mu} \rho \frac{k^{2}}{\epsilon}, \quad C_{\mu}=0.09 .
$$

The viscosity functions $\mu_{k}$ and $\mu_{\epsilon}$ reads (see [20]):

$$
\mu_{k}=\mu+\frac{\mu_{t}}{\sigma_{k}}, \quad \mu_{\epsilon}=\mu+\frac{\mu_{t}}{\sigma_{\epsilon}}, \quad \sigma_{k}=1, \quad \sigma_{\epsilon}=1.3
$$

As usual, $q$ denotes the heat conduction and $p^{\star}=p+\frac{2}{3} \rho k$ the total pressure. The constants $C_{\epsilon 1}, C_{\epsilon 2}, C_{g 1}$ and $C_{g 2}$ are prescribed by the model (Mohammadi and Pironneau [20] propose $C_{\epsilon 1}=1.44$ and $C_{\epsilon 2}=1.92$ while Laborde [16] propose $C_{g 1}=1$ and $C_{g 2}=2$ ). The functions $\rho D>0$ and $\rho D_{t}>0$ denote diffusive coefficients issued from the Fick laws. Finally, $S_{c t}$ denotes the turbulent Schmidt number, $\lambda_{t}=\mu_{t} C_{p} / P_{r t}$ the dynamical turbulent conductivity with $C_{p}$ the specific heat and $P_{r t}=0.9$ the turbulent Prandtl number.

Concerning the combustion, the quantities $\overline{\dot{\omega}_{j}}$ and $\overline{\dot{\omega}_{j} C_{j}^{\prime \prime}}$ are source terms (they do not involve partial derivative of the unknowns) and are given by the PDF model (see Baurle and Girimaji [2] for further details). For the sake of simplicity, we assume that $\overline{\dot{\omega}_{j}}$ are the only terms which couple the unknowns $k_{h}$ and $Q$ with the hydrodynamic equations. In the present work, the perfect gas law is thus assumed:

$$
p=(\gamma-1)\left(E-\rho \frac{u^{2}}{2}-\rho k\right), \quad \gamma \in(1,3] .
$$

We refer the reader to Berthon and Coquel [3] for extensions to more general pressure laws.

Let us recall that the source terms $\overline{\dot{\omega}}_{j}$, given in $[2,16]$, must satisfy: $\sum_{1 \leq j \leq N} \overline{\dot{\omega}_{j}}=0$.

The aim of the present work is to develop a Riemann solver consistent (in a sense to be specified) with the convection system extracted from (1). Unfortunately, the first order extracted system cannot be recast in conservation form since the products $\rho k \partial_{x} u$ and $\rho \epsilon \partial_{x} u$, involved respectively in the $\rho k$ and $\rho \epsilon$ evolution 
equations, never read under divergence form. The nonexistence of a conservation form is proved in Berthon and Coquel [4]. After the work of Dal Maso, LeFloch and Murat [11], Colombeau, Leroux, Noussair and Perrot [7], Raviart and Sainsaulieu [21], the weak solutions of such a system may not be defined in the sense of distributions as soon as the solution admits discontinuities. However, Raviart and Sainsaulieu [21] define the discontinuous solutions as the limit of viscous shock layers of an associated convection-diffusion system when the diffusion tends to zero. These solutions are thus shown to be dependent on the choice of the diffusion operator. In fact, the solutions are dependent on the shape of the diffusion and not on the Reynolds number. Put in other words, the convection and diffusion operators cannot be discretized independently. In the frame of (1), the diffusion operator is prescribed by the model.

The dissipation operator is associated with the convection terms while the Fourier laws and the source terms are dropped into a second step. Such a splitting, according to a physical point of view, yields to remarks of practical interest. First, in a number of models close to (1), the main modeling assumptions coincide with distinct choices of both turbulent heat conduction and source terms. For instance, we refer to [20] where the authors prove that the $(k, \epsilon),(k, l)$ and $(k, \omega)$ models differ solely by the turbulent heat conduction terms (up to a change of variables). As a consequence of such a splitting, the convection-dissipation operator is the same for a class of models while the turbulent conduction operator differs from one model to other. In addition, the splitting makes the flow variables (here $\left.\rho C_{j}, \rho u, E, \rho k, \rho \epsilon\right)$ independent of the passive scalars $\left(\rho k_{h}\right.$ and $\left.\rho Q\right)$. Indeed, the interactions are, generally, governed by the source terms. This last remark finds a numerical interest and it will be used with benefit in the sequel. We adopt the same decomposition and we focus our attention on the convection-dissipation part of (1). The system under consideration thus reads:

$$
\begin{aligned}
& \left\{\begin{array}{l}
\partial_{t} \rho C_{j}+\partial_{x} \rho C_{j} u=\partial_{x}\left(\left(\rho D+\rho D_{t}\right) \partial_{x} C_{j}\right), \quad 1 \leq j \leq N, \\
\partial_{t} \rho u+\partial_{x}\left(\rho u^{2}+p+\frac{2}{3} \rho k\right)=\partial_{x}\left(\left(\mu+\mu_{t}\right) \partial_{x} u\right), \\
\partial_{t} E+\partial_{x}\left(E+p+\frac{2}{3} \rho k\right) u=\partial_{x}\left(\left(\mu+\mu_{t}\right) u \partial_{x} u\right), \\
\partial_{t} \rho k+\partial_{x} \rho k u+\frac{2}{3} \rho k \partial_{x} u=\mu_{t}\left(\partial_{x} u\right)^{2}, \\
\partial_{t} \rho \epsilon+\partial_{x} \rho \epsilon u+\frac{2}{3} C_{\epsilon 1} \rho \epsilon \partial_{x} u=C_{\epsilon 1} \frac{\epsilon}{k} \mu_{t}\left(\partial_{x} u\right)^{2}, \\
\partial_{t} \rho k_{h}+\partial_{x} \rho k_{h} u=\partial_{x}\left(\frac{\lambda_{t}}{C_{p}} \partial_{x} k_{h}\right)+2 \frac{\lambda_{t}}{C_{p}}\left(\partial_{x} p^{\star}\right)^{2}, \\
\partial_{t} \rho Q+\partial_{x} \rho Q u=\partial_{x}\left(\rho D_{t} \partial_{x} Q\right)+2 \frac{\lambda_{t}}{S_{c t}} \sum_{1 \leq j \leq N}\left(\partial_{x} C_{j}\right)^{2}, \\
\left\{\begin{array}{l}
\sum^{\star}=p+\frac{2}{3} \rho k, \\
p=(\gamma-1)\left(E-\rho \frac{u^{2}}{2}-\rho k\right)
\end{array}\right.
\end{array}\right.
\end{aligned}
$$

In order to ensure the definition of LeFloch [19], Raviart and Sainsaulieu [21] for the weak solutions of the first order extracted system, the viscous shock layers, namely traveling wave solutions, of the above system (3) must be proved to exist (see Berthon and Coquel [3,4], Raviart and Sainsaulieu [21], Sainsaulieu [23] for further 
details). We just recall that a traveling wave solution of (3) is a particular solution in the form $\mathbf{v}(x, t)=\widehat{\mathbf{v}}(x-\sigma t)$ with

$$
\lim _{\xi \rightarrow-\infty} \widehat{\mathbf{v}}(\xi)=\mathbf{v}_{L}, \quad \lim _{\xi \rightarrow+\infty} \widehat{\mathbf{v}}(\xi)=\mathbf{v}_{R}, \quad \xi=x-\sigma t
$$

where the triple $\left(\sigma ; \mathbf{v}_{L}, \mathbf{v}_{R}\right)$ is prescribed. We have used the notation $\mathbf{v}$ to denote the unknown vector. These solutions characterize the viscous shock layers and tend, as the diffusion goes to zero, to the expected discontinuous solutions. In the present work, we conjecture existence of traveling wave solutions, but we recall that the result of existence is proved in $[3,4]$ in the framework of the $(k, \epsilon)$ model, i.e. for the sub-system $(3 \mathrm{a})$.

\subsection{Algebraic properties}

Before we present the discrete scheme of the system (3), we need to precise some algebraic properties of (3). We also emphasize inconsistencies of usual finite volume schemes when extended to system in non-conservation form.

First, we establish basic properties satisfied by the convection system extracted from (3). The following straightforward result is given with no proof:

Lemma 1.1. The first order system extracted from (3) is hyperbolic. It admits $u \pm c$ and $u$ as eigenvalues where we have set

$$
c^{2}=\frac{\gamma p}{\rho}+\frac{10}{9} k
$$

The multiplicity of the eigenvalue $u$ is $N+4$ and is associated with a linearly degenerate field, while the multiplicity of the eigenvalues $u \pm c$ is one and associated with genuinely nonlinear fields. Moreover, $u$ and $p^{*}=p+\frac{2}{3} \rho k$ are continuous through the contact discontinuity.

We go on while establishing additional governing equations satisfied by the smooth solutions of (3). These additional laws will be crucial in the next sections devoted to the numerical approximation of the solution of (3). We now state:

Lemma 1.2. The smooth solutions of (3) satisfy in addition the following balance equations:

$$
\begin{aligned}
& \partial_{t} \rho s+\partial_{x} \rho s u=\mu \frac{\gamma-1}{\rho^{\gamma-1}} f^{\prime}\left(f^{-1}(s)\right)\left(\partial_{x} u\right)^{2}, \\
& \partial_{t} \rho s_{t}+\partial_{x} \rho s_{t} u=\mu_{t} \frac{\gamma_{t}-1}{\rho^{\gamma_{t}-1}} f_{t}^{\prime}\left(f_{t}^{-1}\left(s_{t}\right)\right)\left(\partial_{x} u\right)^{2},
\end{aligned}
$$

where $f$ and $f_{t}$ denote smooth invertible functions and the entropies $s$ and $s_{t}$ are defined by:

$$
\begin{aligned}
s & =f\left(\frac{p}{\rho^{\gamma}}\right) \text { and } s_{t}=f_{t}\left(\frac{\left(\gamma_{t}-1\right) \rho k}{\rho^{\gamma_{t}}}\right), \\
\gamma_{t} & =\frac{5}{3} .
\end{aligned}
$$

As a consequence, the smooth solutions of (3) obey

$$
\mu \frac{\gamma-1}{\rho^{\gamma-1}} f^{\prime}\left(f^{-1}(s)\right)\left\{\partial_{t} \rho s_{t}+\partial_{x} \rho s_{t} u\right\}-\mu_{t} \frac{\gamma_{t}-1}{\rho^{\gamma_{t}-1}} f_{t}^{\prime}\left(f_{t}^{-1}\left(s_{t}\right)\right)\left\{\partial_{t} \rho s+\partial_{x} \rho s u\right\}=0 .
$$

The smooth solutions of (3) also satisfy the following balance equation:

$$
\partial_{t} \rho \frac{C_{j}^{2}}{2}+\partial_{x} \rho \frac{C_{j}^{2}}{2} u=\partial_{x}\left(\left(\rho D+\rho D_{t}\right) C_{j} \partial_{x} C_{j}\right)-\left(\rho D+\rho D_{t}\right)\left(\partial_{x} C_{j}\right)^{2},
$$


for all $1 \leq j \leq N$. Finally, the smooth solutions of (3) satisfy in addition the following conservation law:

$$
\partial_{t} \rho \frac{k^{C_{\epsilon 1}}}{\epsilon}+\partial_{x} \rho \frac{k^{C_{\epsilon 1}}}{\epsilon} u=0 .
$$

Since the convexity of the entropies turns out to be crucial in the next section (to establish stability results of the numerical method), the function $f$ and $f_{t}$ have been introduced in the balance equations (6) and (7). In the sequel, the following condition is assumed to be satisfied by both $f$ and $f_{t}$ :

(H) $\quad g \in \mathrm{C}^{2}\left(\mathbb{R}_{+}, \mathbb{R}_{+}\right)$is a strictly decreasing function and satisfies:

$$
\frac{g^{\prime \prime}(x)}{g^{\prime}(x)}<-\frac{\alpha-1}{\alpha} x, \quad \forall x>0, \alpha=\max \left(\gamma, \gamma_{t}\right) .
$$

As soon as the functions $f$ and $f_{t}$, respectively introduced in (6) and (7), satisfy (H), both maps

$$
\begin{aligned}
& (\rho, \rho u, E, \rho s) \rightarrow\left\{\rho s_{t}\right\}(\rho, \rho u, E, \rho s), \\
& \left(\rho, \rho u, E, \rho s_{t}\right) \rightarrow\{\rho s\}\left(\rho, \rho u, E, \rho s_{t}\right),
\end{aligned}
$$

are convex. Let us note from now on that the numerical stability results (namely Th. 2.3 and Th. 2.4) hold false as soon as both above maps are not assumed convex.

Proof of Lemma 1.2. As usual, the momentum equation can be developed to obtain

$$
\partial_{t} u+u \partial_{x} u+\frac{1}{\rho} \partial_{x}\left(p+\frac{2}{3} \rho k\right)=\frac{1}{\rho} \partial_{x}\left(\left(\mu+\mu_{t}\right) \partial_{x} u\right),
$$

so that we deduce

$$
\partial_{t} \rho \frac{u^{2}}{2}+\partial_{x} \rho \frac{u^{2}}{2} u+u \partial_{x}\left(p+\frac{2}{3} \rho k\right)=u \partial_{x}\left(\left(\mu+\mu_{t}\right) \partial_{x} u\right) .
$$

Now, we subtract (12) and the $\rho k$ equation from the total energy equation:

$$
\begin{aligned}
\left\{\partial_{t} E+\partial_{x}\left(E+p+\frac{2}{3} \rho k\right) u\right\} & -\left\{\partial_{t} \rho \frac{u^{2}}{2}+\partial_{x} \rho \frac{u^{2}}{2}+u \partial_{x}\left(p+\frac{2}{3} \rho k\right)\right\} \\
& -\left\{\partial_{t} \rho k+\partial_{x} \rho k u+\frac{2}{3} \rho k \partial_{x} u\right\}=\partial_{x}\left(\left(\mu+\mu_{t}\right) u \partial_{x} u\right)-u \partial_{x}\left(\left(\mu+\mu_{t}\right) \partial_{x} u\right)-\mu_{t}\left(\partial_{x} u\right)^{2},
\end{aligned}
$$

to obtain after computations:

$$
\partial_{t} p+u \partial_{x} p+\gamma p \partial_{x} u=(\gamma-1) \mu\left(\partial_{x} u\right)^{2}
$$

Since

we have:

$$
\partial_{t} \rho+u \partial_{x} \rho+\rho \partial_{x} u=0
$$

$$
\frac{1}{\rho^{\gamma}}\left\{\partial_{t} p+u \partial_{x} p+\gamma p \partial_{x} u\right\}-\frac{\gamma p}{\rho^{\gamma+1}}\left\{\partial_{t} \rho+u \partial_{x} \rho+\rho \partial_{x} u\right\}=\mu \frac{\gamma-1}{\rho^{\gamma}}\left(\partial_{x} u\right)^{2},
$$

which reads

$$
\partial_{t} \frac{p}{\rho^{\gamma}}+u \partial_{x} \frac{p}{\rho^{\gamma}}=\mu \frac{\gamma-1}{\rho^{\gamma}}\left(\partial_{x} u\right)^{2},
$$


to obtain

$$
\partial_{t} s+u \partial_{x} s=\mu \frac{\gamma-1}{\rho^{\gamma}} f^{\prime}\left(f^{-1}(s)\right)\left(\partial_{x} u\right)^{2}
$$

Since we have

$$
\rho\left\{\partial_{t} s+u \partial_{x} s\right\}+s\left\{\partial_{t} \rho+\partial_{x} \rho u\right\}=\partial_{t} \rho s+\partial_{x} \rho s u
$$

the identity (6) is thus obtained. In a same way, we have:

$$
\frac{2 / 3}{\rho^{5 / 3}}\left\{\partial_{t} \rho k+u \partial_{x} \rho k+\frac{5}{3} \rho k \partial_{x} u\right\}-\frac{5 / 3}{\rho^{5 / 3+1}} \frac{2}{3} \rho k\left\{\partial_{t} \rho+u \partial_{x} \rho+\rho \partial_{x} u\right\}=\mu_{t} \frac{5 / 3-1}{\rho^{5 / 3}}\left(\partial_{x} u\right)^{2},
$$

and $(7)$ is thus obtained as soon as $(13)$ is multiplied by $f_{t}^{\prime}\left(\frac{2 / 3 \rho k}{\rho^{5 / 3}}\right)$.

The identity (10) is nothing but

$$
C_{j}\left\{\partial_{t} \rho C_{j}+\partial_{x} \rho C_{j} u\right\}=C_{j} \partial_{x}\left(\left(\rho D+\rho D_{t}\right) \partial_{x} C_{j}\right)
$$

since we have

$$
\partial_{t} \rho C_{j}+\partial_{x} \rho C_{j} u=\rho\left(\partial_{t} C_{j}+u \partial_{x} C_{j}\right)
$$

To obtain the additional conservation law (11), we first note the following identities:

$$
\begin{aligned}
\partial_{t} \rho X+\partial_{x} \rho X u & =\rho\left\{\partial_{t} X+u \partial_{x} X\right\}+X\left\{\partial_{t} \rho+\partial_{x} \rho u\right\} \\
& =\rho\left\{\partial_{t} X+u \partial_{x} X\right\}
\end{aligned}
$$

where $X$ respectively denotes $k$ or $\epsilon$. Then we deduce from the $\rho k$ and $\rho \epsilon$ equations:

$$
\begin{aligned}
& \partial_{t} k+u \partial_{x} k+\frac{2}{3} k \partial_{x} u=\frac{\mu_{t}}{\rho}\left(\partial_{x} u\right)^{2} \\
& \partial_{t} \epsilon+u \partial_{x} \epsilon+\frac{2}{3} C_{\epsilon 1} \epsilon \partial_{x} u=c_{\epsilon 1} \frac{\mu_{t}}{\rho} \frac{\epsilon}{k}\left(\partial_{x} u\right)^{2},
\end{aligned}
$$

to obtain:

$$
C_{\epsilon 1} \frac{\epsilon}{k}\left\{\partial_{t} k+u \partial_{x} k+\frac{2}{3} k \partial_{x} u\right\}-\left\{\partial_{t} \epsilon+u \partial_{x} \epsilon+\frac{2}{3} C_{\epsilon 1} \epsilon \partial_{x} u\right\}=0
$$

which reads

$$
\partial_{t} \frac{k^{C_{\epsilon 1}}}{\epsilon}+u \partial_{x} \frac{k^{C_{\epsilon 1}}}{\epsilon}=0
$$

The last identity (11) is thus proved.

The balance equations (6) and (7) and the conservation law (11) can be proved to be the only non trivial additional equations for smooth solutions. As a consequence and besides several close relationships with the usual Navier-Stokes system, the very discrepancy stays in the lack of $N+6$ non trivial conservation laws. Indeed and without restrictive modeling assumptions, none of the equations (6), (7) and (9) recast as a conservation law except in some special cases which are not of interest. As a consequence, (3) cannot be recast, generally speaking, in full conservation form. 

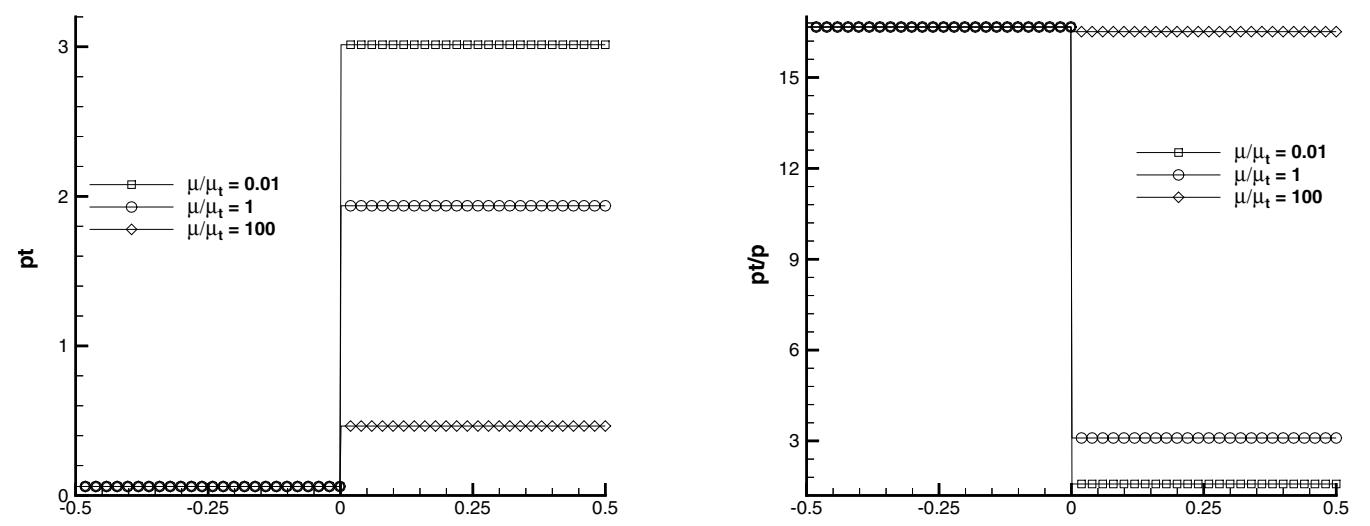

FigURE 1. Viscous shock layers as a function of $\mu / \mu_{t}$ for a given $\left(\sigma, \mathbf{v}_{L}\right)$.

After the pioneering works by LeFloch [19], Raviart-Sainsaulieu [21] and Sainsaulieu [23], the non conservation form met by (3) makes the end states of viscous shock layers to intrinsically depend on the closure relations for the transport coefficients $\mu, \mu_{t}, \lambda_{t}$ and $C_{p}$. Under the assumption of two constant viscosities, such a dependence more precisely occurs in term of the ratio of $\mu$ and $\mu_{t}$ when computing $p$ and $\rho k$. In order to assess this issue, let us focus our attention on the non standard balance equation (9). Its straightforward derivation reflects a cancellation property. Namely the entropy balance equations (6) are not independent but actually evolve proportionally to the ratio of the two viscosities $\mu$ and $\mu_{t}$. Indeed and at least formally, (9) yields once integrated with respect to the space variable:

$$
\frac{\mu_{t}}{\mu+\mu_{t}}\left\{\int_{\mathbb{R}} \frac{\gamma_{t}-1}{\rho^{\gamma_{t}-1}} f_{t}^{\prime}\left(f_{t}^{-1}\left(s_{t}\right)\right)\left(\partial_{t} \rho s+\partial_{x} \rho s u\right) \mathrm{d} x\right\}-\frac{\mu}{\mu+\mu_{t}}\left\{\int_{\mathbb{R}} \frac{\gamma-1}{\rho^{\gamma-1}} f^{\prime}\left(f^{-1}(s)\right)\left(\partial_{t} \rho s_{t}+\partial_{x} \rho s_{t} u\right) \mathrm{d} x\right\}=0,
$$

so that the evolution in time of the two entropies must be kept in balance according to the ratio of the two viscosities. Let us emphasize that by contrast with (6) or (7) where entropy dissipation rates are actually independently imposed, the weighted equation (9) exhibits a compared rate of both entropy dissipations.

The dependence we have just pointed out is numerically illustrated in Figure 1. For a given left end state $\mathbf{v}_{L}$ and a given velocity $\sigma$, the required right end states $\mathbf{v}_{R}$ are defined when solving numerically the nonlinear ODE's system governing traveling wave solutions (see [4]) for various ratios of the viscosities.

\subsection{Negative numerical consequences}

Now, let us deduce from the above section, the main numerical consequences. First, we note that the system (3) involves two distinct types of non-conservation product (NCP): the first order NCP where the product involves a function of the unknowns and one partial derivative of the unknowns (the product $\rho k \partial_{x} u$ for instance) and the second order NCP where two partial derivatives of the unknowns are involved $\left(\mu_{t}\left(\partial_{x} u\right)^{2}\right.$ for instance).

The classical finite volume methods are shown to converge to wrong function in the scalar case by HouLeFloch [15] when approximating first order NCPs. In Berthon and Coquel [5], the failure is shown in the framework of system in non-conservation form (see Fig. 2). The system (3) involves two distinct first order NCPs, namely $\frac{2}{3} \rho k \partial_{x} u$ and $\frac{2}{3} C_{\epsilon 1} \rho \epsilon \partial_{x} u$ respectively in the evolution equations of $\rho k$ and $\rho \epsilon$. However, by virtue of Lemma 1.2, the governing equation of $\rho k$ and $\rho \epsilon$ may be substituted by (7) and (11) to obtain an equivalent system with no first order NCP but for one more second order NCP.

The second order NCPs are shown to be crucial in the characterization of the viscous shock layers (see Berthon and Coquel [5], Berthon, Coquel and LeFloch [6]). Unfortunately, both above papers show that classical schemes largely under resolve these second order NCP (see Fig. 3). In fact, this problem is well-known in the framework 
density

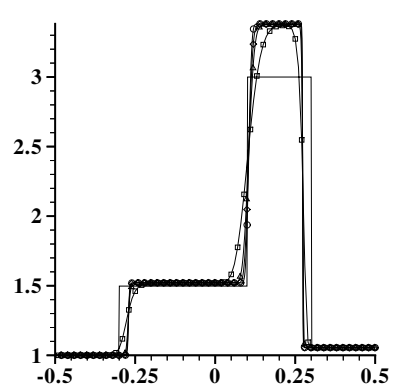

velocity

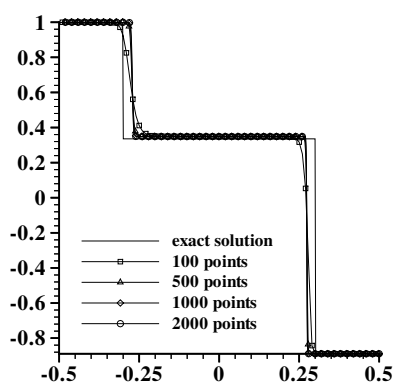

pressure

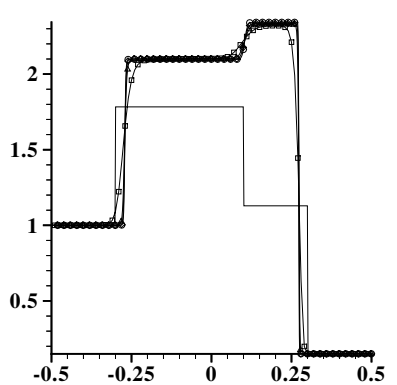

turbulent energy

FIGURE 2. Typical failure of classical finite volume methods in the capture of viscous shock layers.
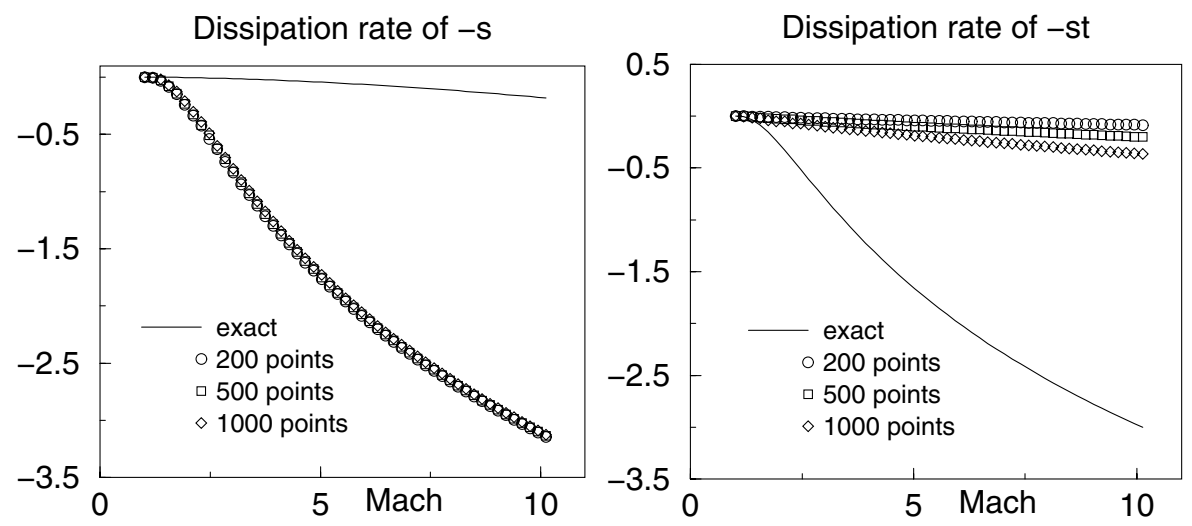

Figure 3. Typical failure when evaluated dissipation rates of entropies $s=\log \left(\frac{p}{\rho^{\gamma}}\right)$ and $s_{t}=$ $\log \left(\frac{\frac{2}{3} \rho k}{\rho^{\gamma t}}\right)$ through a viscous 1 -shock.

of the Navier-Stokes equations when considering the specific entropy balance equation:

$$
\partial_{t} \rho s+\partial_{x} \rho s u=\mu \frac{\gamma-1}{\rho^{\gamma-1}}\left(\partial_{x} u\right)^{2},
$$

instead of the usual conservation law of the total energy.

In the next section, devoted to the numerical approximation of solutions of (3), we briefly recall the nonlinear projection method (see [5]) which ensures stability and agreement with exact solutions in the framework of some non-conservation system such as the $(k, \epsilon)$ model. This method will be shown to be a way to approximate some second order NCPs, but not for all second order NCP. Indeed, as proposed in [5], the nonlinear projection method cannot be extended to resolve the second order NCPs involved in the governing equations of $\rho k_{h}$ and $\rho Q$. This mentioned work indeed deals with the second order NCPs $\left(\partial_{x} u\right)^{2}$. The nonlinear procedure is based on the cancellation property of the entropy dissipation rates, detailed in (6) and (7), to obtain (9) which does not involve second order NCP. Such a property never arises when considering the product $\left(\partial_{x} p^{\star}\right)^{2}$ of the evolving combustion equations. With the assumption of a large Reynolds number, we propose an approximate nonlinear projection method to assess this issue. 


\section{A Godunov type method}

This section is devoted to a nonstandard finite volume method to approximate the solutions of the nonconservation system (3). To assess this issue, let us first note that (3) may be split in two sub-system associated with the turbulence (3a) and the combustion (3b) respectively. Indeed, the system (3a) is independent of (3b) while $(3 \mathrm{~b})$ is coupled with (3a) via the second order terms and the velocity. In the sequel the functions $f$ and $f_{t}$ are fixed according to assumption $(\mathrm{H})$.

\subsection{Numerical approximation of (3a): Turbulence}

In the first step, the solutions of (3a) are approximated. For the sake of simplicity in the following developments, the viscosity functions are assumed to be constant in the sequel. The reader is referred to Section 2.1.4 for the case of varying coefficients.

To reduce the number of NCPs involved in $(3 \mathrm{a})$, we propose to consider the following system, equivalent to (3a) by virtue of Lemma 1.2, where we have substituted both $\rho k$ and $\rho \epsilon$ governing equations by the additional laws (7) and (11) satisfied by the solutions of (3):

$$
\left\{\begin{array}{l}
\partial_{t} \rho C_{j}+\partial_{x} \rho C_{j} u=\partial_{x}\left(\left(\rho D+\rho D_{t}\right) \partial_{x} C_{j}\right), \quad 1 \leq j \leq N, \\
\partial_{t} \rho u+\partial_{x}\left(\rho u^{2}+p+\frac{2}{3} \rho k\right)=\partial_{x}\left(\left(\mu+\mu_{t}\right) \partial_{x} u\right), \\
\partial_{t} E+\partial_{x}\left(E+p+\frac{2}{3} \rho k\right) u=\partial_{x}\left(\left(\mu+\mu_{t}\right) u \partial_{x} u\right), \\
\partial_{t} \rho s_{t}+\partial_{x} \rho s_{t} u=\mu_{t} \frac{\gamma_{t}-1}{\rho^{\gamma_{t}-1}} f_{t}^{\prime}\left(f_{t}^{-1}\left(s_{t}\right)\right)\left(\partial_{x} u\right)^{2}, \\
\partial_{t} \rho \frac{k^{C_{\epsilon 1}}}{\epsilon}+\partial_{x} \rho \frac{k^{C_{\epsilon 1}}}{\epsilon} u=0
\end{array}\right.
$$

with in addition the following balance entropy law:

$$
\partial_{t} \rho s+\partial_{x} \rho s u=\mu \frac{\gamma-1}{\rho^{\gamma-1}} f^{\prime}\left(f^{-1}(s)\right)\left(\partial_{x} u\right)^{2} .
$$

When considering the specific case $N=1$ (or equivalently $C_{1}=1$ ), the system (15) is nothing but the multientropies Navier-Stokes system proposed by [5] where the authors develop a nonlinear projection method to approximate solutions. We adopt this numerical method and we briefly recall the main steps and properties, its extension to the case $N \geq 2$ being obvious.

Let $\Delta t$ and $\Delta x$ respectively denote the time and space increments, chosen to be constant without restriction. The numerical approximate solution, $\mathbf{w}_{h}: \mathbb{R} \times \mathbb{R}_{+} \rightarrow \Omega$, is as usual supposed to be piecewise constant and we set using classical notations:

$$
\mathbf{w}_{h}(x, t)=\mathbf{w}_{i}^{n}, \quad(x, t) \in\left(x_{i-1 / 2}, x_{i+1 / 2}\right) \times\left(t^{n}, t^{n+1}\right), \quad i \in \mathbb{Z}, n \in \mathbb{N},
$$

where the notation $\mathbf{w}$ denotes the unknown vector $\left(\rho C_{1}, \ldots, \rho C_{N}, \rho u, E, \rho s_{t}, \rho \frac{k^{C_{\epsilon 1}}}{\epsilon}\right)$. 


\subsubsection{First step: Extracted first order system $\left(t^{n} \rightarrow t^{n+1,=}\right)$}

Assume that the discrete solution $\mathbf{w}_{h}\left(x, t^{n}\right)$ is known at the time level $t^{n}$. In order to evolve it in time, we propose to solve as a first step the following Cauchy problem:

$$
\left\{\begin{array}{l}
\partial_{t} \rho C_{j}+\partial_{x} \rho C_{j} u=0, \quad 1 \leq j \leq N \\
\partial_{t} \rho u+\partial_{x}\left(\rho u^{2}+p+\frac{2}{3} \rho k\right)=0 \\
\partial_{t} E+\partial_{x}\left(E+p+\frac{2}{3} \rho k\right) u=0 \\
\partial_{t} \rho s_{t}+\partial_{x} \rho s_{t} u=0 \\
\partial_{t} \rho \frac{k^{C_{\epsilon 1}}}{\epsilon}+\partial_{x} \rho \frac{k^{C_{\epsilon 1}}}{\epsilon} u=0
\end{array}\right.
$$

when prescribing the initial data to $\mathbf{w}_{h}\left(x, t^{n}\right) \in \Omega$ where

$$
\begin{aligned}
\Omega=\left\{\left(\rho C_{1}, \ldots, \rho C_{N}, \rho u, E, \rho s_{t}, \rho \frac{k^{C_{\epsilon 1}}}{\epsilon}\right) \in \mathbb{R}^{N+4} ;\right. \\
\rho>0, C_{j} \in[0,1], \sum_{1 \leq j \leq N} C_{j}=1, \rho u \in \mathbb{R}, \\
\left.E-\frac{(\rho u)^{2}}{2 \rho}-\frac{2}{3} \rho^{5 / 3} f_{t}^{-1}\left(s_{t}\right)>0, \rho s_{t}>0, \rho \frac{k^{C_{\epsilon 1}}}{\epsilon}>0\right\} .
\end{aligned}
$$

Weak solutions of the above hyperbolic system are asked to satisfy the following Lax entropy inequality

$$
\partial_{t} \rho S+\partial_{x} \rho S u \leq 0, \quad S=\phi\left(\frac{p}{\rho^{\gamma}}\right)
$$

where $\phi \in \mathrm{C}^{2}(\mathbb{R}, \mathbb{R})$ denotes any function which satisfies $(\mathrm{H})$ to ensure $\mathbf{w} \rightarrow \phi\left(p / \rho^{\gamma}\right)$ convex, to rule out unphysical solutions. For convenience in the discussion, the problem $(18,19)$ is solved exactly. Then under the following CFL like condition:

$$
\frac{\Delta t}{\Delta x} \max \left|\lambda_{i}(\mathbf{w})\right| \leq \frac{1}{2}
$$

the solution is classically made of neighboring and non interacting elementary Riemann solutions. This solution is then classically averaged over each cell (see Godlewski and Raviart [13], Harten, Lax and Van Leer [14]). Let denote by $\mathbf{g}: \Omega \times \Omega \rightarrow \mathbb{R}^{4}$ the associated Lipschitz continuous numerical flux function. Setting $\mathbf{g}_{i+1 / 2}^{n}=$ $\mathbf{g}\left(\mathbf{w}_{i}^{n}, \mathbf{w}_{i+1}^{n}\right)$, the updated solution then reads:

$$
\mathbf{w}_{i}^{n+1,=}=\mathbf{w}_{i}^{n}-\frac{\Delta t}{\Delta x}\left\{\mathbf{g}_{i+1 / 2}^{n}-\mathbf{g}_{i-1 / 2}^{n}\right\}, \quad i \in \mathbb{Z} .
$$

From now on, we state the following maximum principle result of the mass fractions, directly deduced from Larrouturou [17]: 
Lemma 2.1. Under the CFL condition (20), the unknowns $\left(C_{j}\right)_{1 \leq j \leq N}$ satisfy $\left(C_{j}\right)_{i}^{n+1,=} \in[0,1]$ for all $1 \leq$ $j \leq N, i \in \mathbb{Z}$, as soon as

$$
\left(\mathbf{g}_{\left\{\rho C_{j} u\right\}}\right)_{i+1 / 2}^{n}=\left(\mathbf{g}_{\{\rho u\}}\right)_{i+1 / 2}^{n} \times \begin{cases}\left(C_{j}\right)_{i}^{n} & \text { if }\left(\mathbf{g}_{\{\rho u\}}\right)_{i+1 / 2}^{n}<0 \\ \left(C_{j}\right)_{i+1}^{n} & \text { if }\left(\mathbf{g}_{\{\rho u\}}\right)_{i+1 / 2}^{n}>0 .\end{cases}
$$

2.1.2. Second step: Diffusion operator $\left(t^{n+1,=} \rightarrow t^{n+1,-}\right)$

The discrete solution $\mathbf{w}_{h}\left(x, t^{n+1,=}\right)$ is next evolved in time to the date $t^{n+1,-}$ when solving with the initial data $\mathbf{w}_{h}\left(x, t^{n+1,=}\right)$ :

$$
\begin{aligned}
\partial_{t} \rho C_{j} & =\partial_{x}\left(\left(\rho D+\rho D_{t}\right) \partial_{x} C_{j}\right), \quad 1 \leq j \leq N, \\
\partial_{t} \rho u & =\partial_{x}\left(\left(\mu+\mu_{t}\right) \partial_{x} u\right), \\
\partial_{t} E & =\partial_{x}\left(\left(\mu+\mu_{t}\right) u \partial_{x} u\right), \\
\partial_{t} \rho s_{t} & =\mu_{t} \frac{\gamma_{t}-1}{\rho^{\gamma_{t}-1}} f_{t}^{\prime}\left(f_{t}^{-1}\left(s_{t}\right)\right)\left(\partial_{x} u\right)^{2}, \\
\partial_{t} \rho \frac{k^{C_{\epsilon 1}}}{\epsilon} & =0 .
\end{aligned}
$$

In that aim, we suggest to adopt the following implicit finite difference scheme:

$$
\begin{aligned}
\left(\rho C_{j}\right)_{i}^{n+1,-} & =\left(\rho C_{j}\right)_{i}^{n+1,=}+\Delta t{\overline{\partial_{x}\left(\left(\rho D+\rho D_{t}\right) \partial_{x} C_{j}\right.}}_{i}^{n+1,-}, \quad 1 \leq j \leq N, \\
(\rho u)_{i}^{n+1,-} & =(\rho u)_{i}^{n+1,=}+\Delta t{\overline{\partial_{x}\left(\left(\mu+\mu_{t}\right) \partial_{x} u_{i}^{n+1,-}\right.}}^{n+1,-}, \\
E_{i}^{n+1,-} & =E_{i}^{n+1,=}+\Delta t{\overline{\partial_{x}\left(\left(\mu+\mu_{t}\right) u \partial_{x} u\right)_{i}^{n}}}^{n+1,}{\overline{\gamma_{t}-1}}_{\rho_{t}^{\prime}\left(f_{t}^{-1}\left(s_{t}\right)\right)\left(\partial_{x} u\right)^{2}}^{n+1,-}, \\
\left(\rho s_{t}\right)_{i}^{n+1,-} & =\left(\rho s_{t}\right)_{i}^{n+1,=}+\Delta t \mu_{t}{ }^{\rho_{t}-1}, \\
\left(\rho \frac{k^{C_{\epsilon 1}}}{\epsilon}\right)_{i}^{n+1,-} & =\left(\rho \frac{k^{C_{\epsilon 1}}}{\epsilon}\right)_{i}^{n+1,=},
\end{aligned}
$$

where we have set:

$$
\begin{aligned}
& {\overline{\partial_{x}\left(\left(\rho D+\rho D_{t}\right) \partial_{x} C_{j}\right)_{i}^{n+1,-}}}^{n+1} \frac{1}{\Delta x^{2}}\left(\left(\rho D+\rho D_{t}\right)_{i+1 / 2}^{n}\left(\frac{\left(\rho C_{j}\right)_{i+1}^{n+1,-}}{\rho_{i+1}^{n+1,-}}-\frac{\left(\rho C_{j}\right)_{i}^{n+1,-}}{\rho_{i}^{n+1,-}}\right)\right. \\
& \left.-\left(\rho D+\rho D_{t}\right)_{i-1 / 2}^{n}\left(\frac{\left(\rho C_{j}\right)_{i}^{n+1,-}}{\rho_{i}^{n+1,-}}-\frac{\left(\rho C_{j}\right)_{i-1}^{n+1,-}}{\rho_{i-1}^{n+1,-}}\right)\right), \\
& {\overline{\partial_{x}\left(\left(\mu+\mu_{t}\right) \partial_{x} u_{i}^{n}\right.}}^{n+1,-}=\frac{\mu+\mu_{t}}{\Delta x^{2}}\left(M^{n} u_{i+1}-2 M^{n} u_{i}+M^{n} u_{i-1}\right), \\
& {\overline{\partial_{x}\left(\mu+\mu_{t}\right) u \partial_{x} u_{i}}}^{n+1,-}=\frac{\mu+\mu_{t}}{2 \Delta x^{2}}\left(\left(M^{n} u_{i+1}\right)^{2}-2\left(M^{n} u_{i}\right)^{2}+\left(M^{n} u_{i-1}\right)^{2}\right),
\end{aligned}
$$

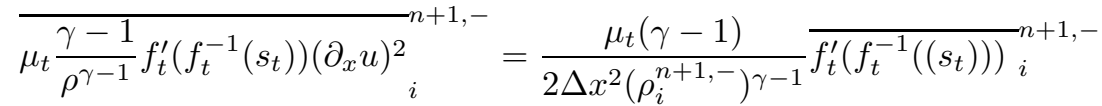

$$
\begin{aligned}
& \times\left(\left(M^{n} u_{i+1}-M^{n} u_{i}\right)^{2}+\left(M^{n} u_{i}-M^{n} u_{i-1}\right)^{2}\right),
\end{aligned}
$$


with $u_{i}=(\rho u)_{i} / \rho_{i}$ and $\left(s_{t}\right)_{i}^{n+1,-}=\left(\rho s_{t}\right)_{i}^{n+1,-} / \rho_{i}^{n+1,-}$ and

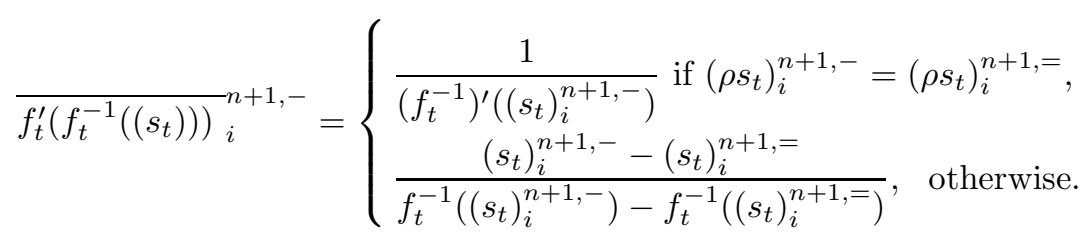

In our setting, the average $\left(\rho D+\rho D_{t}\right)_{i+1 / 2}^{n}$ remains free. To fix the ideas, we will set $\left(\rho D+\rho D_{t}\right)_{i+1 / 2}^{n}=$ $\left(\left(\rho D+\rho D_{t}\right)_{i}^{n}+\left(\rho D+\rho D_{t}\right)_{i+1}^{n}\right) / 2$. In $(24), M^{n}$ denotes a time averaging operator given by:

$$
M^{n} X=\frac{X^{n+1,-}+X^{n+1,=}}{2} .
$$

Let us emphasize that the first three finite difference operators, (24a, 24b) and (24c), preserve by construction the conservation property to be satisfied by the unknowns $\rho C_{j}, \rho u$ and $E$.

Besides and as pointed out below, the benefit of these formulas is twofold.

On the one hand, straightforward computations yield from the above formulas the following identity:

$$
\{\rho s\}\left(\mathbf{w}_{i}^{n+1,-}\right)=\{\rho s\}\left(\mathbf{w}_{i}^{n+1,=}\right)+\Delta t \mu{\overline{\frac{\gamma-1}{\rho^{\gamma-1}} f^{\prime}\left(f^{-1}(s)\right)\left(\partial_{x} u\right)^{2}}}_{i}^{n+1,-},
$$

where ${\overline{\mu \frac{\gamma-1}{\rho^{\gamma-1}} f^{\prime}\left(f^{-1}(s)\right)\left(\partial_{x} u\right)^{2}}}_{i}^{n+1,-}$ is defined by the analogous formula (24d). Hence during the second step (and only the second step), the proposed finite difference operators allow for preserving at the discrete level the expected dissipation rate of the entropy $\rho s$. Other finite difference formulas are actually possible but up to our knowledge, such formulas systematically produce errors in the discrete entropy balance equation (26) for $\rho s$.

On the second hand, the density is kept constant during this second step. Indeed, we establish

Lemma 2.2. The formula (24a) yields the following identity:

$$
\sum_{1 \leq j \leq N}{\overline{\partial_{x}\left(\left(\rho D+\rho D_{t}\right) \partial_{x} C_{j}\right.}}_{i}^{n+1,-}=0,
$$

thus $\rho_{i}^{n+1,-}=\rho_{i}^{n+1=}$. During the second step, the mass fractions $\left(\rho C_{j}\right)_{i}^{n+1,-}$ are solutions of the following linear problems:

$$
\begin{aligned}
\left(\rho C_{j}\right)_{i}^{n+1,-}= & \left(\rho C_{j}\right)_{i}^{n+1,=}+\frac{\Delta t}{\Delta x^{2}}\left(\left(\rho D+\rho D_{t}\right)_{i+1 / 2}^{n}\left(\frac{\left(\rho C_{j}\right)_{i+1}^{n+1,-}}{\rho_{i+1}^{n+1,=}}-\frac{\left(\rho C_{j}\right)_{i}^{n+1,-}}{\rho_{i}^{n+1,=}}\right)\right. \\
& \left.-\left(\rho D+\rho D_{t}\right)_{i-1 / 2}^{n}\left(\frac{\left(\rho C_{j}\right)_{i}^{n+1,-}}{\rho_{i}^{n+1,=}}-\frac{\left(\rho C_{j}\right)_{i-1}^{n+1,-}}{\rho_{i-1}^{n+1,=}}\right)\right)
\end{aligned}
$$

for $1 \leq j \leq N$. The maximum principle $\left(C_{j}\right)_{i}^{n+1,-} \in[0,1]$ is satisfied.

As a consequence, the implicit equations (23b) stay completely decoupled from the others, namely (23c) and $(23 \mathrm{~d})$.

Therefore solving (23) just amounts in practice to evaluate the mass fractions next to invert a positive definite symmetric matrix for the unknown $M^{n} u$. The unknowns $E_{i}^{n+1,-}$ and $\left(\rho s_{t}\right)_{i}^{n+1,-}$ can be then evaluated.

Proof of Lemma 2.2. Since $\rho_{i}^{n+1,-}:=\sum_{1 \leq j \leq N}\left(\rho C_{j}\right)_{i}^{n+1,-}$, the identity (27) arises when summing the identities (24a) for $1 \leq j \leq N$. As a consequence, we have $\rho_{i}^{n+1,-}=\rho_{i}^{n+1,=}$. The linear problems (28) thus hold 
as $\rho_{i}^{n+1,-}$ are substituted by $\rho_{i}^{n+1,=}$ in the operators (24a). Since the matrix to invert is a M-matrix, the maximum principle is obtained. Indeed, let us set

$$
\alpha_{i+1}=\frac{\Delta t}{\Delta x^{2}}\left(\rho D+\rho D_{t}\right)_{i+1 / 2}^{n}>0,
$$

to rewrite $(28)$ in the following form:

$$
-\alpha_{i-1}\left(C_{j}\right)_{i-1}^{n+1,-}+\left(1+\alpha_{i+1}+\alpha_{i-1}\right)\left(C_{j}\right)_{i}^{n+1,-}-\alpha_{i+1}\left(C_{j}\right)_{i+1}^{n+1,-}=\left(C_{j}\right)_{i}^{n+1,=},
$$

where $\left(C_{j}\right)_{i}^{n+1,=} \in[0,1]$. The proof is thus completed.

The above two steps yield a standard finite volume method for approximating the solutions of (15). Such a method will be referred in the sequel as to a classical $\mathrm{L}^{2}$-projection method. Nevertheless, this scheme is easily seen to yield the following discrete form for (9):

$$
\begin{aligned}
& \mu_{t} \frac{\gamma_{t}-1}{\left(\rho_{i}^{n+1,-}\right)^{\gamma_{t}-1}} f_{t}^{\prime}\left(f_{t}^{-1}\left(\left(s_{t}\right)_{i}^{n+1,-}\right)\right)\left(\{\rho s\}\left(\mathbf{w}_{i}^{n+1,-}\right)-(\rho s)_{i}^{n}+\frac{\Delta t}{\Delta x}\left\{\{\rho s u\}_{i+1 / 2}^{n}-\{\rho s u\}_{i-1 / 2}^{n}\right\}\right) \\
& \quad-\mu \frac{\gamma-1}{\left(\rho_{i}^{n+1,-}\right)^{\gamma-1}} f^{\prime}\left(f^{-1}\left(s\left(\mathbf{w}_{i}^{n+1,-}\right)\right)\right)\left(\left(\rho s_{t}\right)_{i}^{n+1,-}-\left(\rho s_{t}\right)_{i}^{n}+\frac{\Delta t}{\Delta x}\left\{\left\{\rho s_{t} u\right\}_{i+1 / 2}^{n}-\left\{\rho s_{t} u\right\}_{i-1 / 2}^{n}\right\}\right)=\mathcal{E}_{i}^{n} \neq 0,
\end{aligned}
$$

where the error $\mathcal{E}_{i}^{n}$ is nothing but the numerical rate of entropy dissipation (see Godlewski and Raviart [13] for further details but also Berthon and Coquel [5]). As usual, we have used the notations $\{\rho s u\}_{i+1 / 2}^{n}$ and $\left\{\rho s_{t} u\right\}_{i+1 / 2}^{n}$ for denoting the associated numerical flux functions.

This strongly implies that the classical $\mathrm{L}^{2}$-projection method can only fail in satisfying the balance entropy condition (9), excepted with violation of the convexity properties of the Lax entropies (see [5]). The reader is referred to the numerical results below for an illustration of the negative consequences of such a failure.

\subsubsection{Third step: Nonlinear projection $\left(t^{n+1,-} \rightarrow t^{n+1}\right)$}

According to the discrete balance equation (29), standard finite volume methods induce a too large numerical rate of entropy dissipation for $\rho s$ in comparison with that of $\rho s_{t}$ that in turn precludes the satisfaction of (9). Here, we propose to add a nonlinear procedure as an additional step to classical $\mathrm{L}^{2}$-projection methods, the so-called nonlinear projection step, which purpose is precisely to correct the former errors. Indeed, the aim of the third step we propose is to redistribute the errors between the two rates of entropy dissipation in order to keep them in balance according to (9). Let us emphasize that the numerical procedure derived below inherits by construction all the desirable stability properties satisfied by relevant approximate Riemann solvers.

In order to preserve the required conservation properties, let us define:

$$
\begin{aligned}
& \left(\rho C_{j}\right)_{i}^{n+1}=\left(\rho C_{j}\right)_{i}^{n+1,-}, 1 \leq j \leq N \\
& (\rho u)_{i}^{n+1}=(\rho u)_{i}^{n+1,-}, \quad E_{i}^{n+1}=E_{i}^{n+1,-}, \quad\left(\rho \frac{k^{C_{\epsilon 1}}}{\epsilon}\right)_{i}^{n+1}=\left(\rho \frac{k^{C_{\epsilon 1}}}{\epsilon}\right)_{i}^{n+1,-} .
\end{aligned}
$$

Then to enforce the validity of the balance entropy condition at the discrete level (9), we propose to seek for $\left(\rho s_{t}\right)_{i}^{n+1}$ as a solution of:

$$
\begin{aligned}
& \mu_{t} \frac{\gamma_{t}-1}{\left(\rho_{i}^{n+1,-}\right)^{\gamma_{t}-1}} f_{t}^{\prime}\left(f_{t}^{-1}\left(\left(s_{t}\right)_{i}^{n+1,-}\right)\right)\left(\{\rho s\}\left(\mathbf{w}_{i}^{n+1}\right)-(\rho s)_{i}^{n}+\frac{\Delta t}{\Delta x}\left\{\{\rho s u\}_{i+1 / 2}^{n}-\{\rho s u\}_{i-1 / 2}^{n}\right\}\right) \\
& -\mu \frac{\gamma-1}{\left(\rho_{i}^{n+1,-}\right)^{\gamma-1}} f^{\prime}\left(f^{-1}\left(\left(s\left(\mathbf{w}_{i}^{n+1,-}\right)\right)\right)\left(\left(\rho s_{t}\right)_{i}^{n+1}-\left(\rho s_{t}\right)_{i}^{n}+\frac{\Delta t}{\Delta x}\left\{\left\{\rho s_{t} u\right\}_{i+1 / 2}^{n}-\left\{\rho s_{t} u\right\}_{i-1 / 2}^{n}\right\}\right)=0\right.
\end{aligned}
$$


where the numerical flux functions $\left\{\rho s_{t} u\right\}_{i+1 / 2}^{n}$ and $\{\rho s u\}_{i+1 / 2}^{n}$ satisfy the Larrouturou formulas [17] (see also [5]). This in turn uniquely defines $(\rho s)_{i}^{n+1}$ according to:

$$
(\rho s)_{i}^{n+1}=\{\rho s\}\left(\left(\rho C_{1}\right)_{i}^{n+1}, \ldots,\left(\rho C_{N}\right)_{i}^{n+1},(\rho u)_{i}^{n+1}, E_{i}^{n+1},\left(\rho s_{t}\right)_{i}^{n+1},\left(\rho \frac{k^{C_{\epsilon 1}}}{\epsilon}\right)_{i}^{n+1}\right)
$$

Numerical methods based on (31) are referred in the sequel as to $\mathrm{L}^{2}$ nonlinear projection methods. The above nonlinear problem in the unknown $\left(\rho s_{t}\right)_{i}^{n+1}$ can be shown to admit a unique nonnegative solution as soon as the approximate Riemann solver involved in the first step 2.1.1 obeys discrete entropy inequalities for the Lax pair $(\rho s, \rho s u)$. Indeed we have (see [5] for the proof):

Theorem 2.3. Under the CFL restriction (20), the $\mathrm{L}^{2}$ nonlinear projection (31) admits a unique solution. This solution satisfy

$$
\{\rho s\}\left(\mathbf{w}_{i}^{n+1}\right)-(\rho s)_{i}^{n}+\frac{\Delta t}{\Delta x}\left\{\{\rho s u\}_{i+1 / 2}^{n}-\{\rho s u\}_{i-1 / 2}^{n}\right\} \leq 0
$$

This above result is completed by the following stability result given in [5]:

Theorem 2.4. Under the CFL restriction (20), the following discrete entropy inequalities are satisfied:

$$
\begin{array}{r}
\{\rho \phi(s)\}\left(\mathbf{w}_{i}^{n+1}\right)-(\rho \phi(s))_{i}^{n}+\frac{\Delta t}{\Delta x}\left\{\{\rho \phi(s) u\}_{i+1 / 2}^{n}-\{\rho \phi(s) u\}_{i-1 / 2}^{n}\right\} \leq 0 \\
\left\{\rho \psi\left(s_{t}\right)\right\}\left(\mathbf{w}_{i}^{n+1}\right)-\left(\rho \psi\left(s_{t}\right)\right)_{i}^{n}+\frac{\Delta t}{\Delta x}\left\{\left\{\rho \psi\left(s_{t}\right) u\right\}_{i+1 / 2}^{n}-\left\{\rho \psi\left(s_{t}\right) u\right\}_{i-1 / 2}^{n}\right\} \leq 0
\end{array}
$$

for any strictly increasing functions $\phi$ and $\psi$ assumed to satisfy the convexity of the maps $\mathbf{w} \rightarrow \rho \phi(s(\mathbf{w}))$ and $\mathbf{w} \rightarrow \rho \psi\left(s_{t}\right)$. The following maximum principles for the specific entropies $s$ and $s_{t}$ are met:

$$
\begin{aligned}
(s)_{i}^{n+1} & \leq \max \left((s)_{i-1}^{n},(s)_{i}^{n},(s)_{i+1}^{n}\right) \\
\left(s_{t}\right)_{i}^{n+1} & \leq \max \left(\left(s_{t}\right)_{i-1}^{n},\left(s_{t}\right)_{i}^{n},\left(s_{t}\right)_{i+1}^{n}\right)
\end{aligned}
$$

The pressure $p\left(\mathbf{w}_{i}^{n+1}\right)$, the kinetic turbulent energy $(\rho k)_{i}^{n+1}$ and its dissipation rate $(\rho \epsilon)_{i}^{n+1}$ stay positive as soon as the density $\rho_{i}^{n+1}$ is positive.

In addition, the following result is deduced from Lemma 2.1 and Lemma 2.2:

Theorem 2.5. Under the CFL restriction (20), the maximum principle

$$
\left(C_{j}\right)_{i}^{n+1} \in[0,1], \quad \text { for all } 1 \leq j \leq N \text { and } i \in \mathbb{Z}
$$

is satisfied.

This concludes the presentation of the nonlinear projection method.

\subsubsection{About variable viscosities}

For the sake of simplicity in the presentation of the nonlinear projection method, actually both viscosities are assumed to be constant. In a more general setting, the viscosities are smooth functions of the unknowns. All the above statements extend easily to such a viscosity function when adopting the following finite difference 
formulas:

$$
\begin{aligned}
& {\overline{\partial_{x}\left(\left(\mu+\mu_{t}\right) \partial_{x} u\right)_{i}^{n+1,-}}}^{n+1}=\frac{1}{\Delta x^{2}}\left(\left(\mu+\mu_{t}\right)_{i+1 / 2}^{n}\left(M^{n} u_{i+1}-M^{n} u_{i}\right)-\left(\mu+\mu_{t}\right)_{i-1 / 2}^{n}\left(M^{n} u_{i}-M^{n} u_{i-1}\right)\right), \\
& {\overline{\partial_{x}\left(\left(\mu+\mu_{t}\right) u \partial_{x} u\right)_{i}^{n+1,-}}}^{n+\frac{1}{2 \Delta x^{2}}}\left(\left(\mu+\mu_{t}\right)_{i+1 / 2}^{n}\left(\left(M^{n} u_{i+1}\right)^{2}-\left(M^{n} u_{i}\right)^{2}\right)\right. \\
& \left.-\left(\mu+\mu_{t}\right)_{i-1 / 2}^{n}\left(\left(M^{n} u_{i}\right)^{2}-\left(M^{n} u_{i-1}\right)^{2}\right)\right) \text {, } \\
& {\overline{\mu_{t} \frac{\gamma_{t}-1}{\rho^{\gamma_{t}-1}} f_{t}^{\prime}\left(f_{t}^{-1}\left(s_{t}\right)\right)\left(\partial_{x} u\right)^{2}}}_{i}^{n+1,-}=\frac{\gamma_{t}-1}{2 \Delta x^{2}\left(\rho_{i}^{n+1,-}\right)^{\gamma_{t}-1}} f_{t}^{\prime}\left(f_{t}^{-1}\left(\left(s_{t}\right)_{i}^{n+1,-}\right)\right) \\
& \times\left(\left(\mu_{t}\right)_{i+1 / 2}^{n}\left(M^{n} u_{i+1}-M^{n} u_{i}\right)^{2}+\left(\mu_{t}\right)_{i-1 / 2}^{n}\left(M^{n} u_{i}-M^{n} u_{i-1}\right)^{2}\right),
\end{aligned}
$$

where $\left(\mu+\mu_{t}\right)_{i+1 / 2}^{n}=(\mu)_{i+1 / 2}^{n}+\left(\mu_{t}\right)_{i+1 / 2}^{n}$. The extension of the nonlinear projection (31) thus reads:

$$
\begin{aligned}
{\overline{\mu_{t}}}_{i}^{n+1,-} & \frac{\gamma_{t}-1}{\left(\rho_{i}^{n+1,-}\right)^{\gamma_{t}-1}} f_{t}^{\prime}\left(f_{t}^{-1}\left(\left(s_{t}\right)_{i}^{n+1,-}\right)\right)\left(\{\rho s\}\left(\mathbf{w}_{i}^{n+1}\right)-(\rho s)_{i}^{n}+\frac{\Delta t}{\Delta x}\left\{\{\rho s u\}_{i+1 / 2}^{n}-\{\rho s u\}_{i-1 / 2}^{n}\right\}\right) \\
& -\bar{\mu}_{i}^{n+1,-} \frac{\gamma-1}{\left(\rho_{i}^{n+1,-}\right)^{\gamma-1}} f^{\prime}\left(f^{-1}\left(\left(s\left(\mathbf{w}_{i}^{n+1,-}\right)\right)\right)\left(\left(\rho s_{t}\right)_{i}^{n+1}-\left(\rho s_{t}\right)_{i}^{n}+\frac{\Delta t}{\Delta x}\left\{\left\{\rho s_{t} u\right\}_{i+1 / 2}^{n}-\left\{\rho s_{t} u\right\}_{i-1 / 2}^{n}\right\}\right)=0\right.
\end{aligned}
$$

where the averaged viscosities are defined as follows:

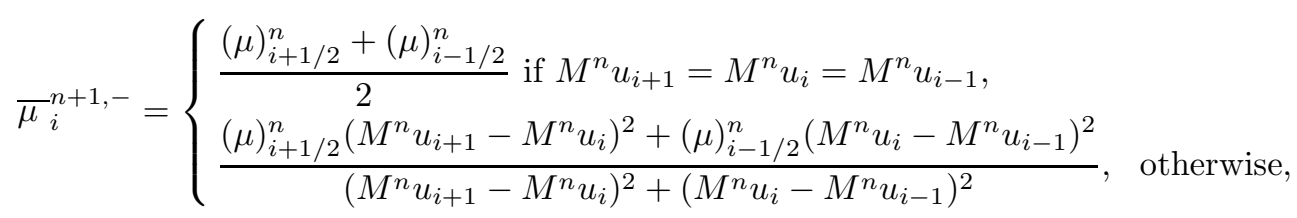

with the analogous formula for the viscosity $\mu_{t}$.

\subsubsection{Nonlinear procedure and second order NCPs}

To conclude the present section, we emphasize that the above nonlinear method can be understood as a systematic procedure to compute the entropy $\rho s_{t}$ dissipation rate, i.e. the second order $\operatorname{NCP}\left(\partial_{x} u\right)^{2}$. Indeed, the nonlinear step (31) reads as follows:

$$
\begin{aligned}
\left(\rho s_{t}\right)_{i}^{n+1} & -\left(\rho s_{t}\right)_{i}^{n}+\frac{\Delta t}{\Delta x}\left\{\left\{\rho s_{t} u\right\}_{i+1 / 2}^{n}-\left\{\rho s_{t} u\right\}_{i-1 / 2}^{n}\right\}=\mu_{t} \frac{\gamma_{t}-1}{\left(\rho_{i}^{n+1,-}\right)^{\gamma_{t}-1}} f_{t}^{\prime}\left(f_{t}^{-1}\left(\left(s_{t}\right)_{i}^{n+1,-}\right)\right) \times \\
& \times\left[\frac{\left(\rho_{i}^{n+1,-}\right)^{\gamma-1}}{(\gamma-1) \mu} \frac{1}{f^{\prime}\left(f^{-1}\left(s\left(\mathbf{w}_{i}^{n+1,-}\right)\right)\right)}\left(\{\rho s\}\left(\mathbf{w}_{i}^{n+1}\right)-(\rho s)_{i}^{n}+\frac{\Delta t}{\Delta x}\left\{\{\rho s u\}_{i+1 / 2}^{n}-\{\rho s u\}_{i-1 / 2}^{n}\right\}\right)\right],
\end{aligned}
$$

which is nothing but a discrete form of (7). When compared with the discrete form of (7) obtained from the first two steps, we deduce from (35) the following formulas:

$$
\begin{aligned}
& {\overline{\left(\partial_{x} u\right)^{2}}}_{i}^{n+1}= \\
& \quad \frac{1}{(\gamma-1) \mu} \frac{1}{f^{\prime}\left(f^{-1}\left(s\left(\mathbf{w}_{i}^{n+1,-}\right)\right)\right)}\left(\frac{\{\rho s\}\left(\mathbf{w}_{i}^{n+1}\right)-(\rho s)_{i}^{n}}{\Delta t}+\frac{1}{\Delta x}\left\{\{\rho s u\}_{i+1 / 2}^{n}-\{\rho s u\}_{i-1 / 2}^{n}\right\}\right) \geq 0 .
\end{aligned}
$$


Put in other words, the discrete formulas of $\left(\partial_{x} u\right)^{2}$, here proposed, to evaluate the dissipation rate of $\rho s_{t}$ are consistent with the dissipation rate of the entropy $\rho s$ since, from (6), we have:

$$
\left(\partial_{x} u\right)^{2}=\frac{\rho^{\gamma-1}}{(\gamma-1) \mu} \frac{1}{f^{\prime}\left(f^{-1}(s)\right)} \times\left(\partial_{t} \rho s+\partial_{x} \rho s u\right),
$$

and (36) is a discrete form of (37).

\subsection{Numerical approximation of (3b): Combustion}

From the numerical approximation of the solutions of the turbulence subsystem (3a), in the sequel the discrete solution $\mathbf{w}_{h}\left(x, t^{n+1}\right)$ is assumed to be known. Now, we deal with the combustion subsystem (3b) setting the same notations as stated in the above section.

Similarly to the approximation of (3a), the discrete vector

$$
\mathbf{u}_{h}\left(x, t^{n}\right)=\left(\left(\rho k_{h}\right)_{h}\left(x, t^{n}\right),(\rho Q)_{h}\left(x, t^{n}\right)\right),
$$

is assumed to be known at the time level $t^{n}$, and is evolved in time by two steps. For the sake of simplicity, let $\lambda_{t}$ and $C_{p}$ be assumed to be constant in the sequel. The extension to variable coefficients is directly deduced from the Section 2.1.4 where an extension to variable viscosities is proposed for the turbulence subsystem.

\subsubsection{First step $\left(t^{n} \rightarrow t^{n+1,-}\right)$}

We propose to solve as a first step the following Cauchy problem:

$$
\left\{\begin{array}{l}
\partial_{t} \rho C_{j}+\partial_{x} \rho C_{j} u=0, \quad 1 \leq j \leq N, \\
\partial_{t} \rho u+\partial_{x}\left(\rho u^{2}+p+\frac{2}{3} \rho k\right)=0 \\
\partial_{t} E+\partial_{x}\left(E+p+\frac{2}{3} \rho k\right) u=0 \\
\partial_{t} \rho s_{t}+\partial_{x} \rho s_{t} u=0 \\
\partial_{t} \rho \frac{k^{C_{\epsilon 1}}}{\epsilon}+\partial_{x} \rho \frac{k^{C_{\epsilon 1}}}{\epsilon} u=0 \\
\partial_{t} \rho k_{h}+\partial_{x} \rho k_{h} u=0 \\
\partial_{t} \rho Q+\partial_{x} \rho Q u=0
\end{array}\right.
$$

when prescribing the initial data to $\mathbf{v}_{h}\left(x, t^{n}\right)=\left(\mathbf{w}_{h}\left(x, t^{n}\right), \mathbf{u}_{h}\left(x, t^{n}\right)\right) \in \Omega \times \mathbb{R}_{+}^{\star} \times \mathbb{R}_{+}^{\star}$. Once again, we solve (38) with the CFL like condition (20). Setting $G_{i+1 / 2}^{n}=G\left(\mathbf{v}_{i}^{n}, \mathbf{v}_{i+1}^{n}\right)$, where $G$ denotes the Lipschitz continuous numerical flux function, the updated solution reads:

$$
\mathbf{v}_{i}^{n+1,-}=\mathbf{v}_{i}^{n}-\frac{\Delta t}{\Delta x}\left\{G_{i+1 / 2}^{n}-G_{i-1 / 2}^{n}\right\}, \quad i \in \mathbb{Z} .
$$

Let us note from now on that the first step in fact reads:

$$
G_{i+1 / 2}^{n}={ }^{t}\left(g_{i+1 / 2}^{n}, \tilde{g}_{i+1 / 2}^{n}\right),
$$


where $g_{i+1 / 2}^{n}$ is defined by (21) and $\tilde{g}_{i+1 / 2}^{n}=\tilde{g}\left(\mathbf{v}_{i}^{n}, \mathbf{v}_{i+1}^{n}\right)$ with $\tilde{g}$ the numerical flux function associated with the subsystem (3b).

The combustion first step is concluded when stated the following easy result:

Lemma 2.6. Under the CFL restriction (20), the combustion unknowns satisfy positiveness: $\left(\rho k_{h}\right)_{i}^{n+1,-}>0$ and $(\rho Q)_{i}^{n+1,-}>0$ for all $i \in \mathbb{Z}$.

We omit the proof which is a direct consequence of the Godunov type method (for instance, see Godlewski and Raviart [13]).

\subsubsection{Second step: Diffusion operator $\left(t^{n+1,-} \rightarrow t^{n+1}\right)$}

The discrete vector $\mathbf{u}_{h}\left(x, t^{n+1,-}\right)$ is next evolved in time to the date $t^{n+1}$ when solving with the initial data $\mathbf{u}_{h}\left(x, t^{n+1,-}\right)$ :

$$
\left\{\begin{array}{l}
\partial_{t} \rho k_{h}=\partial_{x}\left(\frac{\lambda_{t}}{C_{p}} \partial_{x} k_{h}\right)+2 \frac{\lambda_{t}}{C_{p}}\left(\partial_{x} p^{\star}\right)^{2} \\
\partial_{t} \rho Q=\partial_{x}\left(\rho D_{t} \partial_{x} Q\right)+2 \frac{\lambda_{t}}{S_{c t}} \sum_{1 \leq j \leq N}\left(\partial_{x} C_{j}\right)^{2}
\end{array}\right.
$$

For that purpose, we suggest to adopt the following implicit difference scheme:

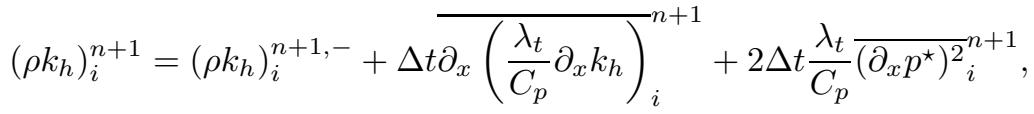

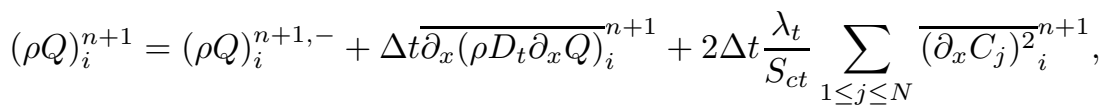

where we have set:

$$
\begin{aligned}
\overline{\partial x}_{x}\left(\frac{\lambda_{t}}{C_{p}} \partial_{x} k_{h}\right)_{i}^{n+1} & =\frac{1}{\Delta x^{2}} \frac{\lambda_{t}}{C_{p}}\left(\frac{\left(\rho k_{h}\right)_{i+1}^{n+1}}{\rho_{i+1}^{n+1}}-2 \frac{\left(\rho k_{h}\right)_{i}^{n+1}}{\rho_{i}^{n+1}}+\frac{\left(\rho k_{h}\right)_{i-1}^{n+1}}{\rho_{i-1}^{n+1}}\right) \\
\overline{\partial x}_{x}\left(\rho D_{t} \partial_{x} Q\right)_{i}^{n+1} & =\frac{1}{\Delta x^{2}}\left[\left(\rho D_{t}\right)_{i+1 / 2}^{n+1}\left(\frac{(\rho Q)_{i+1}^{n+1}}{\rho_{i+1}^{n+1}}-\frac{(\rho Q)_{i}^{n+1}}{\rho_{i}^{n+1}}\right)-\left(\rho D_{t}\right)_{i-1 / 2}^{n+1}\left(\frac{(\rho Q)_{i}^{n+1}}{\rho_{i}^{n+1}}-\frac{(\rho Q)_{i-1}^{n+1}}{\rho_{i-1}^{n+1}}\right)\right] .
\end{aligned}
$$

To complete the implicit difference scheme (42), we have to choose formulas for the discrete form of the dissipation rate $\left(\partial_{x} p^{\star}\right)^{2}$ and $\left(\partial_{x} C_{j}\right)^{2}$. As exemplified in the next section, basic finite difference formulas induce large errors for the approximated solutions when compared either the exact solutions. Indeed, basic formulas predict too low dissipation rates (see Fig. 3) which are of prime importance in the capture of viscous shock layers (see [5] to further details).

\subsubsection{Discrete form of $\left(\partial_{x} C_{j}\right)^{2}$}

Concerning $\left(\partial_{x} C_{j}\right)^{2}$, the dissipation rate of the $\rho Q$ governing equation is actually independently imposed. In fact, the evolution in time of both $\rho Q$ and $\left(\rho C_{j}^{2} / 2\right)_{1 \leq j \leq N}$ must be kept in balance according to the additional law (10). Since we have

$$
\left(\partial_{x} C_{j}\right)^{2}=-\frac{1}{\rho D+\rho D_{t}}\left\{\partial_{t} \rho \frac{C_{j}^{2}}{2}+\partial_{x} \rho \frac{C_{j}^{2}}{2} u-\partial_{x}\left(\left(\rho D+\rho D_{t}\right) C_{j} \partial_{x} C_{j}\right)\right\},
$$


for all $1 \leq j \leq N$, we thus propose the following formula:

$$
\begin{aligned}
&{\overline{\left(\partial_{x} C_{j}\right)^{2}}}_{i}^{n+1}=-\frac{1}{\left(\rho D+\rho D_{t}\right)_{i}^{n+1}} \\
& \times\left\{\frac{\left\{\rho \frac{C_{j}^{2}}{2}\right\}\left(\mathbf{w}_{i}^{n+1}\right)-\left(\rho \frac{C_{j}^{2}}{2}\right)_{i}^{n}}{\Delta t}+\frac{\left\{\rho \frac{C_{j}^{2}}{2} u\right\}_{i+1 / 2}^{n}-\left\{\rho \frac{C_{j}^{2}}{2} u\right\}_{i-1 / 2}^{n}}{\Delta x}-{\overline{\partial_{x}\left(\left(\rho D+\rho D_{t}\right) C_{j} \partial_{x} C_{j}\right)_{i}}}_{i+1}\right\}
\end{aligned}
$$

where we have set

$$
\begin{aligned}
& {\overline{\partial_{x}\left(\left(\rho D+\rho D_{t}\right) C_{j} \partial_{x} C_{j}\right)_{i}^{n+1}}}^{n+1}=\frac{1}{2 \Delta x^{2}}\left(\left(\rho D+\rho D_{t}\right)_{i+1 / 2}^{n}\left(\left(\left(C_{j}\right)_{i+1}^{n+1}\right)^{2}-\left(\left(C_{j}\right)_{i}^{n+1}\right)^{2}\right)\right. \\
& \left.-\left(\rho D+\rho D_{t}\right)_{i-1 / 2}^{n}\left(\left(\left(C_{j}\right)_{i}^{n+1}\right)^{2}-\left(\left(C_{j}\right)_{i-1}^{n+1}\right)^{2}\right)\right),
\end{aligned}
$$

where $C_{j}:=\left(\rho C_{j}\right) / \rho$.

Let us emphasize from now on that the above two steps yield, at the discrete level, the following balance equation deduced from (10) and the $\rho Q$ equation:

$$
\begin{aligned}
& \left(\rho D+\rho D_{t}\right)\left\{\partial_{t} \rho Q+\partial_{x} \rho Q u-\partial_{x}\left(\rho D_{t} \partial_{x} Q\right)\right\} \\
& +2 \frac{\lambda_{t}}{S_{c t}} \sum_{1 \leq j \leq N}\left\{\partial_{t} \rho \frac{C_{j}^{2}}{2}+\partial_{x} \rho \frac{C_{j}^{2}}{2} u-\partial_{x}\left(\left(\rho D+\rho D_{t}\right) C_{j} \partial_{x} C_{j}\right)\right\}=0
\end{aligned}
$$

As a consequence, we deal with the dissipation rate $\left(\partial_{x} C_{j}\right)^{2}$ as a nonlinear projection method, similar to the procedure (31), with the benefit of the splitting turbulence-combustion making explicit the nonlinear procedure (45).

The discrete form of the mass fraction variance equation is thus obtained when establishing the following positiveness result:

Lemma 2.7. Under the CFL restriction (20), $(\rho Q)_{i}^{n+1} \geq 0$ for all $i \in \mathbb{Z}$.

Since the matrix to be inverted to solve (42b) is monotone, the above result readily follows from Lemma 2.6 (see also the proof of the Lem. 2.2) and

Lemma 2.8. Let assume ${\overline{\left(\partial_{x} C_{j}\right)^{2}}}_{i}^{n+1}$ be given by (45) for all $1 \leq j \leq N$, then ${\overline{\left(\partial_{x} C_{j}\right)^{2}}}_{i}^{n+1} \geq 0$ for all $i \in \mathbb{Z}$.

Proof. First, let us recall that $\left(\rho C_{j}\right)_{i}^{n+1,=}$ is defined by $(21),\left(\rho C_{j}\right)_{i}^{n+1,-}$ is defined by $(23 \mathrm{a})$ and $\left(\rho C_{j}\right)_{i}^{n+1}=$ $\left(\rho C_{j}\right)_{i}^{n+1,-}$ and $\rho_{i}^{n+1}=\rho_{i}^{n+1,-}$ from (30) while $\rho_{i}^{n+1,-}=\rho_{i}^{n+1,=}$ by virtue of Lemma 2.2. Since the function $\left(\rho, \rho C_{j}\right) \rightarrow \rho C_{j}^{2} / 2$ is convex, the following inequality easily follows using standard arguments (see for instance Godlewski-Raviart [13], Tadmor [24], Coquel-Perthame [10]):

$$
\left\{\rho \frac{C_{j}^{2}}{2}\right\}\left(\mathbf{w}_{i}^{n+1,=}\right)-\left(\rho \frac{C_{j}^{2}}{2}\right)_{i}^{n}+\frac{\Delta t}{\Delta x}\left\{\left\{\rho \frac{C_{j}^{2}}{2} u\right\}_{i+1 / 2}^{n}-\left\{\rho \frac{C_{j}^{2}}{2} u\right\}_{i-1 / 2}^{n}\right\}=-\left(\tilde{\mathcal{E}}_{j}\right)_{i}^{n} \leq 0
$$

which is a direct consequence of the well-known Jensen inequality.

Now, let us consider (23a) to write

$$
\left(\frac{C_{j}}{2}\right)_{i}^{n+1,-} \times\left(\rho C_{j}\right)_{i}^{n+1,-}=\left(\frac{C_{j}}{2}\right)_{i}^{n+1,-} \times\left(\rho C_{j}\right)_{i}^{n+1,=}+\left(\frac{C_{j}}{2}\right)_{i}^{n+1,-} \times \Delta t{\overline{\partial_{x}\left(\left(\rho D+\rho D_{t}\right) \partial_{x} C_{j}\right)_{i}^{n+1,-}}}^{n}
$$


which reads, after a straightforward computation, as follows:

$$
\begin{aligned}
\left\{\rho \frac{C_{j}^{2}}{2}\right\}\left(\mathbf{w}_{i}^{n+1,-}\right)= & \left\{\rho \frac{C_{j}^{2}}{2}\right\}\left(\mathbf{w}_{i}^{n+1,=}\right)-\frac{\rho_{i}^{n+1}}{2}\left(\left(C_{j}\right)_{i}^{n+1,-}-\left(C_{j}\right)_{i}^{n+1,=}\right)^{2} \\
& +\frac{\left(C_{j}\right)_{i}^{n+1,-}}{2}\left(\left(\rho C_{j}\right)_{i}^{n+1,-}-\left(\rho C_{j}\right)_{i}^{n+1,=}\right)+\frac{\Delta t}{2}\left(C_{j}\right)_{i}^{n+1,-} \frac{\partial_{x}\left(\left(\rho D+\rho D_{t}\right) \partial_{x} C_{j}\right)_{i}^{n+1,-}}{} .
\end{aligned}
$$

Since

$$
\left(\left(\rho C_{j}\right)_{i}^{n+1,-}-\left(\rho C_{j}\right)_{i}^{n+1,=}\right)=\Delta t{\bar{\partial} x\left(\left(\rho D+\rho D_{t}\right) \partial_{x} C_{j}\right)_{i}^{n+1,-}}^{n}
$$

where we have

$$
\begin{aligned}
\left(C_{j}\right)_{i}^{n+1,-} & {\overline{\partial_{x}\left(\left(\rho D+\rho D_{t}\right) \partial_{x} C_{j}\right)_{i}^{n+1,-}}}^{n+1}={\overline{\partial_{x}\left(\left(\rho D+\rho D_{t}\right) C_{j} \partial_{x} C_{j}\right)_{i}}}_{i}^{n+1,-} \\
& -\frac{1}{2 \Delta x^{2}}\left[\left(\rho D+\rho D_{t}\right)_{i+1 / 2}^{n}\left(\left(C_{j}\right)_{i+1}^{n+1,-}-\left(C_{j}\right)_{i}^{n+1,-}\right)^{2}+\left(\rho D+\rho D_{t}\right)_{i-1 / 2}^{n}\left(\left(C_{j}\right)_{i}^{n+1,-}-\left(C_{j}\right)_{i-1}^{n+1,-}\right)^{2}\right]
\end{aligned}
$$

using $\left(\rho C_{j}\right)_{i}^{n+1}=\left(\rho C_{j}\right)_{i}^{n+1,-}$ and $\rho_{i}^{n+1}=\rho_{i}^{n+1,-}$, we obtain

$$
\begin{aligned}
& \frac{\left\{\rho \frac{C_{j}^{2}}{2}\right\}\left(\mathbf{w}_{i}^{n+1}\right)-\left(\rho \frac{C_{j}^{2}}{2}\right)_{i}^{n}}{\Delta t}+\frac{\left\{\rho \frac{C_{j}^{2}}{2} u\right\}_{i+1 / 2}^{n}-\left\{\rho \frac{C_{j}^{2}}{2} u\right\}_{i-1 / 2}^{n}}{\Delta x}-\bar{\partial}_{x}\left(\left(\rho D+\rho D_{t}\right) C_{j} \partial_{x} C_{j}\right)_{i}^{n+1}= \\
& -\left(\tilde{\mathcal{E}}_{j}\right)_{i}^{n}-\frac{\rho_{i}^{n+1}}{2}\left(\left(C_{j}\right)_{i}^{n+1,-}-\left(C_{j}\right)_{i}^{n+1,=}\right)^{2} \\
& -\frac{1}{2 \Delta x^{2}}\left[\left(\rho D+\rho D_{t}\right)_{i+1 / 2}^{n}\left(\left(C_{j}\right)_{i+1}^{n+1,-}-\left(C_{j}\right)_{i}^{n+1,-}\right)^{2}+\left(\rho D+\rho D_{t}\right)_{i-1 / 2}^{n}\left(\left(C_{j}\right)_{i}^{n+1,-}-\left(C_{j}\right)_{i-1}^{n+1,-}\right)^{2}\right] \leq 0 .
\end{aligned}
$$

The proof is thus completed.

\subsubsection{Discrete form of $\left(\partial_{x} p^{\star}\right)^{2}$}

Once again, the dissipation rate of the $\rho k_{h}$ equation, namely $\left(\partial_{x} p^{\star}\right)^{2}$, is actually independently imposed and once again basic finite difference formulas of $\left(\partial_{x} p^{\star}\right)^{2}$ induce large errors. The very discrepancy between above dissipation rates $\left(\partial_{x} u\right)^{2}$ and $\left(\partial_{x} C_{j}\right)^{2}$ stays in the lack of a balance equation which prescribes the discrete formula to be used.

After the recent work of Coquel and LeFloch [8] (but see also [6] in the framework of systems in conservation form), dissipation rates are numerically shown to be negligible far away from viscous shock layers for relevant large enough Reynolds number as soon as dissipation rates vanish through contact waves (through which $u$ and $p+\frac{2}{3} \rho k$ are continuous).

Put in other words, we have $\left(\partial_{x} p^{\star}\right)^{2}:=O(1 / R e)$, but also $\left(\partial_{x} u\right)^{2}:=O(1 / R e)$, far away from shock layers. Of course and as emphasized in the above sections, this turns out to be very different through a viscous shock layer where $\left(\partial_{x} p^{\star}\right)^{2}$ plays a major role.

When characterized through a viscous shock layer, the dissipation rate $\left(\partial_{x} p^{\star}\right)^{2}$ is shown to be evolved proportionally to $\left(\partial_{x} u\right)^{2}$ as follows:

$$
\left(\partial_{x} p^{\star}\right)^{2}=\Theta(\mathbf{v})\left(\partial_{x} u\right)^{2}, \quad \text { in a shock layer, }
$$

where $\Theta$ denotes a function to be specified. As a consequence, we have:

$$
\partial_{t} \rho k_{h}+\partial_{x} \rho k_{h} u=\partial_{x}\left(\frac{\lambda_{t}}{C_{p}} \partial_{x} k_{h}\right)+2 \frac{\lambda_{t}}{C_{p}} \Theta(\mathbf{v})\left(\partial_{x} u\right)^{2}+O\left(\frac{1}{R e}\right),
$$


to obtain an approximate balance equation, deduced from (37):

$$
\left\{\partial_{t} \rho k_{h}+\partial_{x} \rho k_{h} u-\partial_{x}\left(\frac{\lambda_{t}}{C_{p}} \partial_{x} k_{h}\right)\right\}-2 \frac{\lambda_{t}}{C_{p}} \Theta(\mathbf{v}) \frac{\rho^{\gamma-1}}{(\gamma-1) \mu} \frac{1}{f^{\prime}\left(f^{-1}(s)\right)}\left\{\partial_{t} \rho s+\partial_{x} \rho s u\right\}=O\left(\frac{1}{R e}\right) .
$$

We thus propose the following formula:

$$
{\overline{\left(\partial_{x} p^{\star}\right)^{2}}}_{i}^{n+1}=\Theta\left(\mathbf{v}_{i}^{n+1}\right){\overline{\left(\partial_{x} u\right)^{2}}}_{i}^{n+1}
$$

where ${\overline{\left(\partial_{x} u\right)^{2}}}_{i}^{n+1}$ is given by (36).

At the end of the second step, (50) is enforced at the discrete level.

To conclude the presentation of the discrete formula of $\left(\partial_{x} p^{\star}\right)^{2}$, the precise from of $(48)$ is established (the proof is given below):

Lemma 2.9. Through a viscous shock layer, the following identity holds true:

$$
\left(\partial_{x} p^{\star}\right)^{2}=\left[\left(1+\frac{\gamma_{t}-1}{\gamma-1} \frac{\mu_{t}}{\mu}\right)\left(\gamma p-\rho \frac{\partial_{x} p}{\partial_{x} \rho}\right)-\left(\gamma p+\frac{2}{3} \gamma_{t} \rho k\right)\right]^{2} \frac{\left(\partial_{x} u\right)^{2}}{(u-\sigma)^{2}}
$$

where $\sigma=\partial_{x}(\rho u) / \partial_{x} \rho$ denotes the propagation speed of the wave.

In (51), since the function $\Theta$ involves derivatives, we need to be more precise in the discrete formula to be used. First, let us recall that never the density stays constant trough a compression wave. Then, we will consider ${\overline{\left(\partial_{x} p^{\star}\right)^{2}}}_{i}^{n+1}=0$ as soon as $\rho_{i+1}^{n+1}=\rho_{i-1}^{n+1}$. Next, for $\rho_{i+1}^{n+1} \neq \rho_{i-1}^{n+1}$ we set

$$
{\frac{\bar{\partial}_{x} p}{\partial_{x}}}_{i}^{n+1}=\frac{p_{i+1}^{n+1}-p_{i-1}^{n+1}}{\rho_{i+1}^{n+1}-\rho_{i-1}^{n+1}} \quad \text { and } \quad \sigma_{i}^{n+1}=\frac{(\rho u)_{i+1}^{n+1}-(\rho u)_{i-1}^{n+1}}{\rho_{i+1}^{n+1}-\rho_{i-1}^{n+1}}
$$

Let us specify the case $u_{i}^{n+1}-\sigma_{i}^{n+1}=0$. Such a situation does not occur in a viscous shock layer (but only for contact wave). As a consequence, if $u_{i}^{n+1}=\sigma_{i}^{n+1}$ we are in a region where $\left(\partial_{x} p^{\star}\right)^{2}=O(1 / R e)$. We thus set ${\overline{\left(\partial_{x} p^{\star}\right)^{2}}}_{i}^{n+1}=0$.

To summarize, the proposed formula reads as follows:

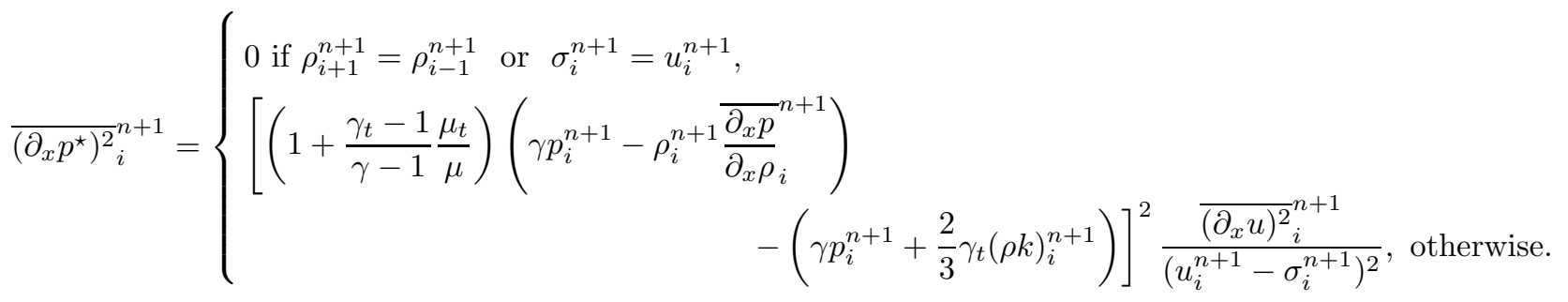

We conclude the discrete form of $\left(\rho k_{h}\right)$ with the following result:

Lemma 2.10. Under the CFL condition (20), $\left(\rho k_{h}\right)_{i}^{n+1} \geq 0$ for all $i \in \mathbb{Z}$.

The proof is a direct consequence of the monotone property of the matrix to be inverted to solve (42a) and the positiveness satisfied by (36) (see also the proof of the Lem. 2.2).

The numerical results which arise when a rarefaction wave is approximated, do not give on coarse meshes a fairly good agreement as expected when compared with the exact solution (briefly detailed in the next section). In fact, the discrete form (51) is based on the numerical dissipation rate of the entropy $\rho s$. This one needs a high level refinement to be small enough (and thus to be negligible) when approximating smooth solutions, 


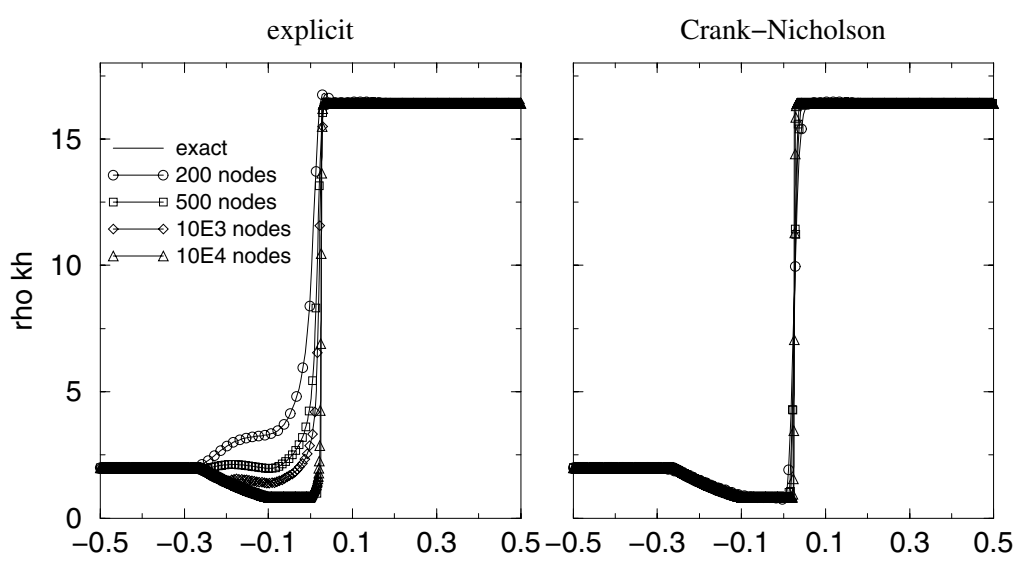

FIGURE 4. Mesh refinement for rarefaction wave using ${\overline{\left(\partial_{x} u\right)^{2}}}_{i}^{n+1}$ and ${\overline{\left(\partial_{x} u\right)^{2}}}_{i}^{n+1 / 2}$.

rarefaction wave for instance. To illustrate our purpose, we display in the Figure 4 the approximated solution of the Riemann problem for (3) with an initial discontinuity located at $x=0$ separating the following left and right states:

$$
\begin{aligned}
& \rho_{L}=1, \quad u_{L}=-1, \quad p_{L}=1, \quad k_{L}=1, \quad\left(k_{h}\right)_{L}=2, \quad Q=1, \\
& \rho_{R}=0.41, \quad u_{R}=0.26, \quad p_{R}=0.29, \quad k_{R}=0.55, \quad\left(k_{h}\right)_{R}=40, \quad Q=1 .
\end{aligned}
$$

The parameters of the model are given by:

$$
\frac{\mu_{t}}{\mu}=1, \quad \frac{\lambda_{t}}{S_{c t}\left(D+D_{t}\right)}=\frac{1}{2}, \quad \frac{\lambda_{t}}{C_{p} \mu}=0.01 .
$$

To enforce a near zero numerical dissipation rate of entropy for smooth solution, we propose a Crank-Nicholson type discrete formula of the numerical entropy flux function and we set:

$$
{\overline{\left(\partial_{x} p^{\star}\right)^{2}}}_{i}^{n+1}=\Theta\left(\mathbf{v}_{i}^{n+1}\right){\overline{\left(\partial_{x} u\right)^{2}}}_{i}^{n+1 / 2}
$$

where

$$
\begin{aligned}
{\overline{\left(\partial_{x} u\right)_{i}^{2}}}_{i}^{n+1 / 2}= & \frac{\left(\rho_{i}^{n+1}\right)^{\gamma-1}}{(\gamma-1) \mu} \frac{1}{f^{\prime}\left(f^{-1}\left(s\left(\mathbf{w}_{i}^{n+1}\right)\right)\right)} \\
& \times\left(\frac{\{\rho s\}\left(\mathbf{w}_{i}^{n+1}\right)-(\rho s)_{i}^{n}}{\Delta t}+\frac{1}{\Delta x}\left\{\frac{\{\rho s u\}_{i+1 / 2}^{n+1}+\{\rho s u\}_{i+1 / 2}^{n}}{2}-\frac{\{\rho s u\}_{i-1 / 2}^{n+1}+\{\rho s u\}_{i-1 / 2}^{n}}{2}\right\}\right)
\end{aligned}
$$

This numerical procedure makes the numerical results to be in agreement with the exact solution for mesh refinements of interest. The "convergence rate" is in fact increased (see Fig. 4).

To conclude the section, we establish the Lemma 2.9 .

Proof of Lemma 2.9. The viscous shock profiles are defined by the traveling wave solutions, i.e. particular solutions in the form $\mathbf{v}(x, t)=\widehat{\mathbf{v}}(x-\sigma t)$ where $\sigma \in \mathbb{R}$ denotes the propagation speed of the wave. For the sake of simplicity, we set $\xi=x-\sigma t$. These solutions satisfy the limits (4). The function $\widehat{\mathbf{v}}$ is a traveling wave 
solution of (3) iff it is a solution of the following ODE system:

$$
\begin{aligned}
& -\sigma d_{\xi} \rho C_{j}+d_{\xi} \rho C_{j} u=d_{\xi}\left(\left(\rho D+\rho D_{t}\right) d_{\xi} C_{j}\right), \quad 1 \leq j \leq N, \\
& -\sigma d_{\xi} \rho u+d_{\xi}\left(\rho u^{2}+p+\frac{2}{3} \rho k\right)=d_{\xi}\left(\left(\mu+\mu_{t}\right) d_{\xi} u\right), \\
& -\sigma d_{\xi} E+d_{\xi}\left(E+p+\frac{2}{3} \rho k\right) u=d_{\xi}\left(\left(\mu+\mu_{t}\right) u d_{\xi} u\right), \\
& -\sigma d_{\xi} \rho k+d_{\xi} \rho k u+\frac{2}{3} \rho k d_{\xi} u=\mu_{t}\left(d_{\xi} u\right)^{2}, \\
& -\sigma d_{\xi} \rho \epsilon+d_{\xi} \rho \epsilon u+\frac{2}{3} C_{\epsilon 1} \rho \epsilon d_{\xi} u=C_{\epsilon 1} \frac{\epsilon}{k} \mu_{t}\left(d_{\xi} u\right)^{2} \\
& -\sigma d_{\xi} \rho k_{h}+d_{\xi} \rho k_{h} u=d_{\xi}\left(\frac{\lambda_{t}}{C_{p}} d_{\xi} k_{h}\right)+2 \frac{\lambda_{t}}{C_{p}}\left(d_{\xi} p^{\star}\right)^{2}, \\
& -\sigma d_{\xi} \rho Q+d_{\xi} \rho Q u=d_{\xi}\left(\rho D_{t} d_{\xi} Q\right)+2 \frac{\lambda_{t}}{S_{c t}} \sum_{1 \leq j \leq N}\left(d_{\xi} C_{j}\right)^{2} .
\end{aligned}
$$

First, the sum of the $N$ mass fractions gives

$$
-\sigma d_{\xi} \rho+d_{\xi} \rho u=0,
$$

to obtain $\sigma=d_{\xi} \rho u / d_{\xi} \rho$, where $d_{\xi} \rho$, and thus $d_{\xi} u$, does not vanish (see $[3,4]$ ).

Since the state law (2) is assumed, we easily deduce from (55c) and (55d) the following identity:

$$
-\sigma d_{\xi} p+d_{\xi} p u+(\gamma-1) p d_{\xi} u=(\gamma-1) \mu\left(d_{\xi} u\right)^{2} .
$$

Now, let us note the following equality:

$$
-\sigma d_{\xi} X+d_{\xi} X u+(\alpha-1) X d_{\xi} u=(u-\sigma) d_{\xi} X+\alpha X d_{\xi} u,
$$

where $\alpha \in \mathbb{R}$ and $X$ denotes respectively $p$ and $\frac{2}{3} \rho k$, to obtain:

$$
\begin{aligned}
& (u-\sigma) d_{\xi} p+\gamma p d_{\xi} u=(\gamma-1) \mu\left(d_{\xi} u\right)^{2}, \\
& (u-\sigma) d_{\xi} \frac{2}{3} \rho k+\gamma_{t}\left(\frac{2}{3} \rho k\right) d_{\xi} u=\left(\gamma_{t}-1\right) \mu_{t}\left(d_{\xi} u\right)^{2} .
\end{aligned}
$$

The sum of the two above identities reads:

$$
(u-\sigma) d_{\xi} p^{\star}=\left[\left((\gamma-1) \mu+\left(\gamma_{t}-1\right) \mu_{t}\right) d_{\xi} u-\left(\gamma p+\frac{2}{3} \gamma_{t} \rho k\right)\right] d_{\xi} u .
$$

From (58), we have

$$
d_{\xi} u=\frac{(u-\sigma) \frac{d_{\xi} p}{d_{\xi} u}+\gamma p}{(\gamma-1) \mu},
$$

where

$$
(u-\sigma)=-\rho \frac{d_{\xi} u}{d_{\xi} \rho}
$$


We thus obtain

$$
d_{\xi} p^{\star}=\left[\left(1+\frac{\gamma_{t}-1}{\gamma-1} \frac{\mu_{t}}{\mu}\right)\left(\gamma p-\rho \frac{d_{\xi} p}{d_{\xi} \rho}\right)-\left(\gamma p+\frac{2}{3} \gamma_{t} \rho k\right)\right] \frac{d_{\xi} u}{u-\sigma},
$$

and the proof is completed.

\section{NUMERICAL EXPERIMENTS}

The ability of the above discussed schemes, namely the $\mathrm{L}^{2}$-projection method and the nonlinear projection scheme, in the capture of viscous shock layers for (3) is evaluated when testing their sensitivity in the prediction of the end states with respect to the mesh refinement when an ideal gas is considered with constant adiabatic exponent for two components $(N=2)$. For the sake of comparison, the systematic approach for approximating the solutions of systems in non conservation form is included in the benchmarks. In what follows, Riemann solutions of (60) are approximated using one of the most widely used method: namely the Roe scheme [22] and its extension to multicomponent flows [1,9]. We have considered an approximate Riemann solver instead of an exact Godunov scheme (as prescribed in the previous section) to emphasize that our procedure turns out to be a systematic correction of the $\mathrm{L}^{2}$-projection method. The theoretical properties, established in the previous section, agree with the stability properties of the adopted finite volume method in the first step. Concerning the boundary conditions, we have just considered boundary conditions according to the system (38) (see [13]). In the following numerical results, the heat conduction is assumed negligible. In the case of non zero heat conduction and turbulent conductivity, actually, the numerical method we propose is based on an additional step where heat conduction is discretized by usual formulas (see [20]).

\subsection{The usual approach for solving (3)}

For the sake of comparison, we end the present section when briefly recalling the most usual (if not systematic) approach for approximating the solutions of systems in non conservation form like (3). According to this approach, all the non conservative products are rejected to the right hand side of the governing equations and are treated as "source terms". The first step is therefore concerned with the following first order extracted system in conservation form:

$$
\left\{\begin{array}{l}
\partial_{t} \rho C_{j}+\partial_{x} \rho C_{j} u=0, \quad 1 \leq j \leq N, \\
\partial_{t} \rho u+\partial_{x}\left(\rho u^{2}+p+\frac{2}{3} \rho k\right)=0 \\
\partial_{t} E+\partial_{x}\left(E+p+\frac{2}{3} \rho k\right) u=0 \\
\partial_{t} \rho k+\partial_{x} \rho k u=0 \\
\partial_{t} \rho \epsilon+\partial_{x} \rho \epsilon u=0 \\
\partial_{t} \rho k_{h}+\partial_{x} \rho k_{h} u=0 \\
\partial_{t} \rho Q+\partial_{x} \rho Q u=0 \\
p=(\gamma-1)\left(E-\rho \frac{u^{2}}{2}-\rho k\right) \\
p^{\star}=p+\frac{2}{3} \rho k \\
\sum_{1 \leq j \leq N} C_{j}=1
\end{array}\right.
$$


while in a next step, the "source terms"

$$
-\frac{2}{3} \rho k \partial_{x} u,-\frac{2}{3} C_{\epsilon 1} \rho \epsilon \partial_{x} u, \mu_{t}\left(\partial_{x} u\right)^{2}, C_{\epsilon 1} \frac{\epsilon}{k} \mu_{t}\left(\partial_{x} u\right)^{2}, 2 \frac{\lambda_{t}}{C_{p}}\left(\partial_{x} p^{\star}\right)^{2}, 2 \frac{\lambda_{t}}{S_{c t}} \sum_{1 \leq j \leq N}\left(\partial_{x} C_{j}\right)^{2}
$$

are to be given some ad hoc finite difference approximations. We refer for instance the reader to Larrouturou and Olivier [18] and Mohammadi and Pironneau [20] concerning the details. The (severe) drawbacks in the resulting numerical schemes are illustrated below. Let us furthermore emphasize after Forestier-Herard-Louis [12] that exact Riemann solutions of (60) all preserve the positiveness of the total pressure $p^{\star}$ (far away from vacuum) but do not necessarily keep non negative the pressure $p$. By contrast, both pressure $p$ and kinetic turbulent energy $\rho k$ in (3) can be shown to stay positive (again far away from vacuum; we do not consider the vacuum problem in the present work). Such schemes are referred to classical methods.

\subsection{Numerical results}

The initial data of the Cauchy problems to be solved are made of two constant states, the discontinuity being located at $x=0$.

The associated exact solutions thus correspond to smooth regularizations of Riemann solutions for the underlying first order system extracted from (3). They are thus made up generally speaking of the juxtaposition of traveling wave solutions and "rarefaction" solutions. We do not develop the way to obtain the exact solutions. But, we just emphasize that the exact solutions are obtained when integrating, using Maple subroutines, the ODE's system (55) for governing traveling wave solutions. After Raviart and Sainsaulieu [21], in the limit of a rescaling parameter, the exact solutions of the Riemann problem for the hyperbolic system (3) compatible with the diffusive tensor are thus obtained.

Discrete solutions are systematically compared with the exact solutions.

All the calculations described below have been performed according to the following strategy. An exact Roe type linearization for system (18) and (38) yields an approximate Riemann solver to solve the first step 2.1.1 and 2.2.1 (see [5,9] for the detailed formulas). Successive grids refinements, ranging from 200 to 1000 cells, are considered. The CFL number is fixed at the constant value 0.5.

Two test cases, labeled A and B, are addressed. The first one is the juxtaposition of two traveling wave with speed $\sigma= \pm 1$, while case B is made of a rarefaction wave and a traveling wave. Here, both viscosities $\mu$ and $\mu_{t}$, both diffusion coefficients $D$ and $D_{t}$, the dynamical turbulent viscosity $\lambda_{t}$ and the specific heat $C_{p}$ are assumed to be fixed positive constants.

In all the benchmarks discussed below, the Reynolds number is set at the constant value Rey $=10^{5}$, while the viscosity ratio $\mu_{t} / \mu$ is set at 1 . Following $[4,5]$, the case of $\mu_{t} / \mu=1$ turns out to be the more difficult test for numerical simulation and we adopt this ratio rather than $\mu_{t} / \mu>>1$. The other coefficients are fixed as follows:

$$
\gamma=7 / 5, \quad \frac{\lambda_{t}}{S_{c t}\left(D+D_{t}\right)}=\frac{1}{2} \text { and } \quad \frac{\lambda_{t}}{C_{p} \mu}= \begin{cases}0.01 & \text { case A } \\ 100 & \text { case B. }\end{cases}
$$

The associated initial data are defined in Table 1. 
TABLE 1. Initial data.

\begin{tabular}{|c|c|c|c|c|c|c|c|c|}
\hline Test & $\rho$ & $u$ & $p$ & $C_{1}$ & $k$ & $\epsilon$ & $k_{h}$ & $\mathrm{Q}$ \\
\hline \multirow{2}{*}{$\mathrm{A}$} & 10 & 0.1 & 1 & 0.7 & 0.01 & 1 & 0 & 1 \\
\cline { 2 - 9 } & 3.9055 & -2.3488 & 0.1044 & 0.2 & 3.0276 & 250.7002 & 4.5544 & 3 \\
\hline \multirow{2}{*}{$\mathrm{B}$} & 1 & 1 & -1 & 0.7 & 1 & 1 & 2 & 1 \\
\cline { 2 - 9 } & 0.0354 & 0.0193 & -2.1027 & 0.2 & 3.1772 & 0.1457 & 47.2714 & 3 \\
\hline
\end{tabular}
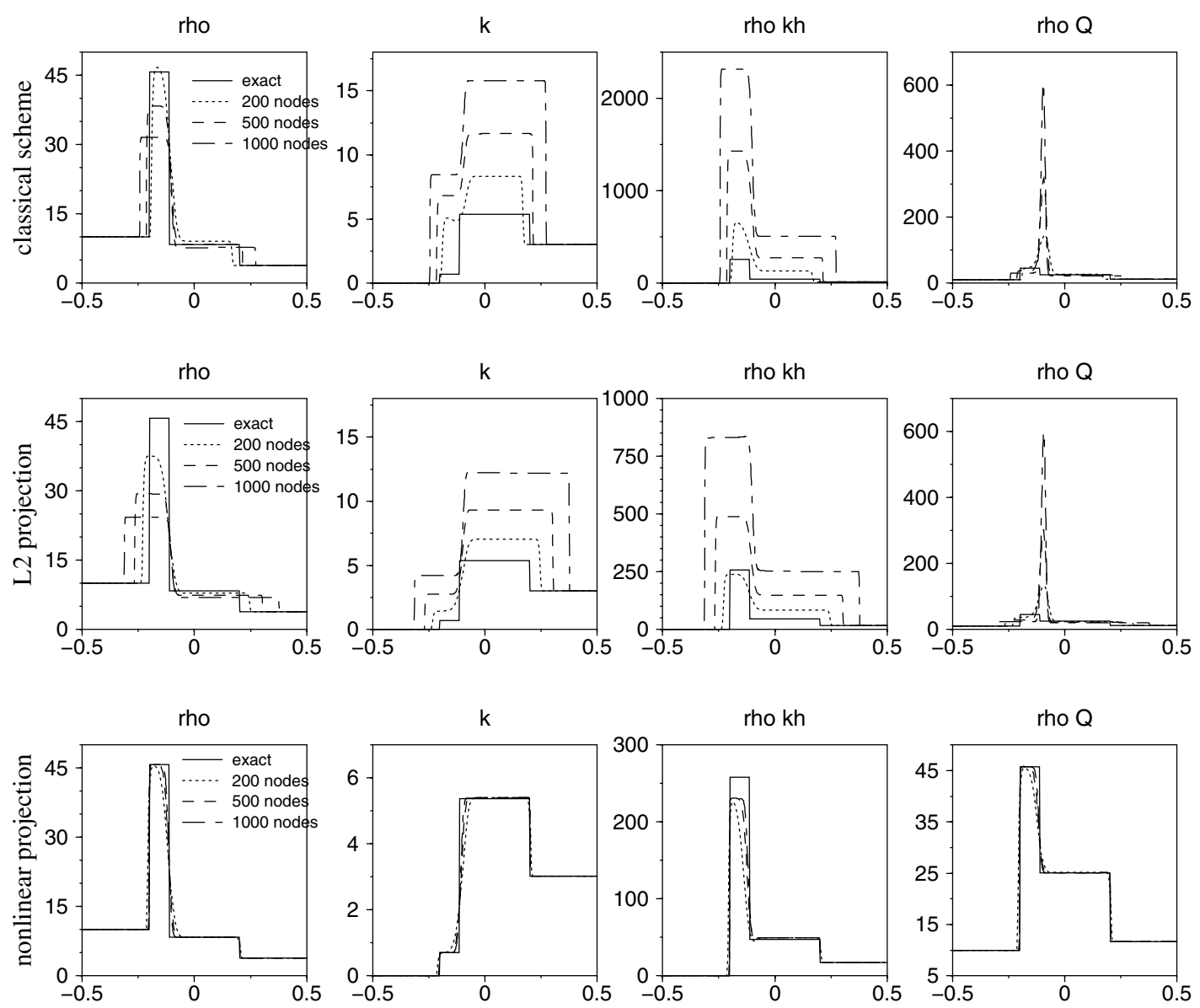

Figure 5. Problem A.

All the figures assess that the usual numerical strategy (see Sect. 3.1) grossly fails to properly restore the correct end states. Considering the $\mathrm{L}^{2}$-projection method, once again large errors occur. These two schemes furthermore suffer from a dramatic sensitivity with respect to mesh refinements and discrete solutions do not seem to converge to a given limit function even for the finest proposed grids. The nonlinear $\mathrm{L}^{2}$-projection 

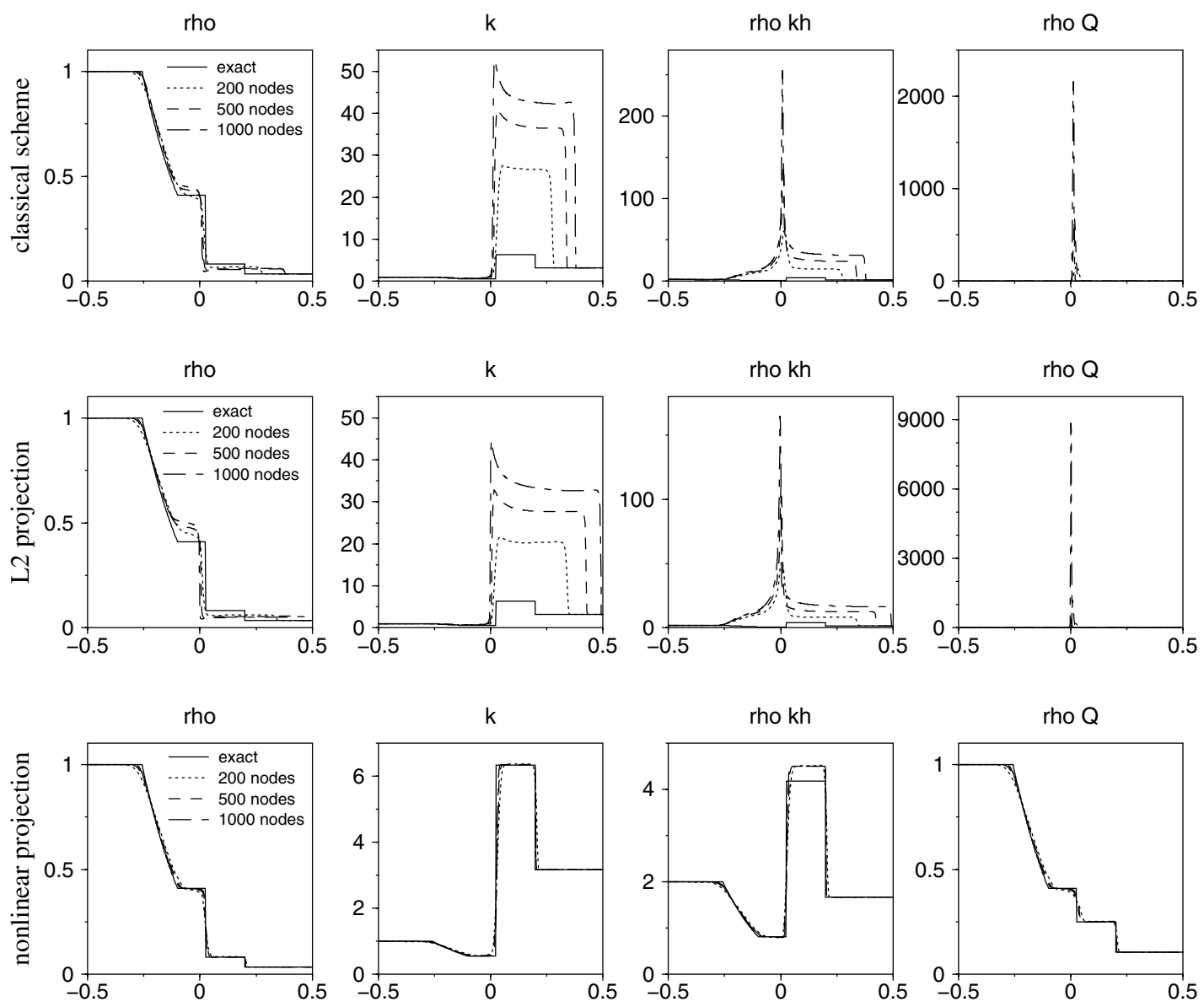

Figure 6. Problem B.

method produces approximate solutions that achieve a fairly good agreement with the exact solutions while staying almost non-sensitive with $\Delta x$ in the two investigated problems. However, we note that the approximation of $\rho k_{h}$, obtained by the nonlinear procedure, involves an error in the prediction of the right state of the 1-shock. We conjecture that this lack of accuracy lies in the numerical dissipation rate of the entropy $s$. Indeed, the production term $\left(\partial_{x} p^{\star}\right)^{2}$ is approximated via the dissipation rate of $s$ but for a discrete formula which involves numerical errors. According to the large errors, the considered scales in Figures 5 and 6 differ with the displayed method.

\section{REFERENCES}

[1] R. Abgrall, An extension of Roe's upwind scheme to algebraic equilibrium real gas models. Comput. and Fluids 19 (1991) $171-182$.

[2] R.A. Baurle and S.S. Girimaji, An assumed PDF Turbulence-Chemistery closure with temperature-composition correlations. 37th Aerospace Sciences Meeting (1999).

[3] C. Berthon and F. Coquel, Travelling wave solutions of a convective diffusive system with first and second order terms in nonconservation form, Hyperbolic problems: theory, numerics, applications, vol. I, Zürich (1998) 47-54, Intern. Ser. Numer. Math. 129 Birkhäuser (1999).

[4] C. Berthon and F. Coquel, About shock layers for compressible turbulent flow models, work in preparation, preprint MAB 01-29 2001 (http://www.math.u-bordeaux.fr/ perthon). 
[5] C. Berthon and F. Coquel, Nonlinear projection methods for multi-entropies Navier-Stokes systems, Innovative methods for numerical solutions of partial differential equations, Arcachon (1998), World Sci. Publishing, River Edge (2002) 278-304.

[6] C. Berthon, F. Coquel and P. LeFloch, Entropy dissipation measure and kinetic relation associated with nonconservative hyperbolic systems (in preparation).

[7] J.F. Colombeau, A.Y. Leroux, A. Noussair and B. Perrot, Microscopic profiles of shock waves and ambiguities in multiplications of distributions. SIAM J. Numer. Anal. 26 (1989) 871-883.

[8] F. Coquel and P. LeFloch, Convergence of finite difference schemes for conservation laws in several space dimensions: a general theory. SIAM J. Numer. Anal. 30 (1993) 675-700.

[9] F. Coquel and C. Marmignon, A Roe-type linearization for the Euler equations for weakly ionized multi-component and multi-temperature gas. Proceedings of the AIAA 12th CFD Conference, San Diego, USA (1995).

[10] F. Coquel and B. Perthame, Relaxation of energy and approximate Riemann solvers for general pressure laws in fluid dynamics. SIAM J. Numer. Anal. 35 (1998) 2223-2249.

[11] G. Dal Maso, P. LeFloch and F. Murat, Definition and weak stability of a non conservative product. J. Math. Pures Appl. 74 (1995) 483-548.

[12] A. Forestier, J.M. Herard and X. Louis, A Godunov type solver to compute turbulent compressible flows. C. R. Acad. Sci. Paris Sér. I Math. 324 (1997) 919-926.

[13] E. Godlewski and P.A. Raviart, Hyperbolic systems of conservations laws. Springer, Appl. Math. Sci. 118 (1996).

[14] A. Harten, P.D. Lax and B. Van Leer, On upstream differencing and Godunov type schemes for hyperbolic conservation laws. SIAM Rev. 25 (1983) 35-61.

[15] T.Y. Hou and P.G. LeFloch, Why nonconservative schemes converge to wrong solutions: error analysis. Math. Comp. 62 (1994) 497-530.

[16] L. Laborde, Modélisation et étude numérique de flamme de diffusion supersonique et subsonique en régime turbulent. Ph.D. thesis, Université Bordeaux I, France (1999).

[17] B. Larrouturou, How to preserve the mass fractions positivity when computing compressible multi-component flows. J. Comput. Phys. 95 (1991) 59-84.

[18] B. Larrouturou and C. Olivier, On the numerical appproximation of the K-eps turbulence model for two dimensional compressible flows. INRIA report, No. 1526 (1991).

[19] P.G. LeFloch, Entropy weak solutions to nonlinear hyperbolic systems under non conservation form. Comm. Partial Differential Equations 13 (1988) 669-727.

[20] B. Mohammadi and O. Pironneau, Analysis of the K-Epsilon Turbulence Model. Masson Eds., Rech. Math. Appl. (1994).

[21] P.A. Raviart and L. Sainsaulieu, A nonconservative hyperbolic system modelling spray dynamics. Part 1. Solution of the Riemann problem. Math. Models Methods Appl. Sci. 5 (1995) 297-333.

[22] P.L. Roe, Approximate Riemann solvers, parameter vectors and difference schemes. J. Comput. Phys. 43 (1981) 357-372.

[23] L. Sainsaulieu, Travelling waves solutions of convection-diffusion systems whose convection terms are weakly nonconservative. SIAM J. Appl. Math. 55 (1995) 1552-1576.

[24] E. Tadmor, A minimum entropy principle in the gas dynamics equations. Appl. Numer. Math. 2 (1986) 211-219.

To access this journal online:

www.edpsciences.org 